\title{
Perturbation theory for the redshift-space matter power spectra after reconstruction
}

\author{
Chiaki Hikage* \\ Kavli Institute for the Physics and Mathematics of the Universe (Kavli IPMU, WPI), \\ University of Tokyo, 5-1-5 Kashiwanoha, Kashiwa, Chiba 277-8583, Japan \\ Kazuya Koyama \\ Institute of Cosmology and Gravitation, University of Portsmouth, Portsmouth PO1 3FX, United Kingdom \\ Ryuichi Takahashi@ \\ Faculty of Science and Technology, Hirosaki University, \\ 3 Bunkyo-cho, Hirosaki, Aomori 036-8588, Japan
}

(Received 15 November 2019; accepted 19 January 2020; published 10 February 2020)

\begin{abstract}
We derive the one-loop perturbative formula of the redshift-space matter power spectrum after density field reconstruction in the Zeldovich approximation. We find that the reconstruction reduces the amplitudes of nonlinear one-loop perturbative terms significantly by partially erasing the nonlinear mode coupling between density and velocity fields. In comparison with $N$-body simulations, we find that both the monopole and quadrupole spectra of reconstructed matter density fields agree with the one-loop perturbation theory up to higher wave number than those before reconstruction. We also evaluate the impact on cosmic growth rate assuming the survey volume and the number density like the Baryon Oscillation Spectroscopic Survey and find that the total error, including statistical and systematic ones due to one-loop approximation, decreases by half.
\end{abstract}

DOI: 10.1103/PhysRevD.101.043510

\section{INTRODUCTION}

Large-scale structure in the Universe is a powerful cosmological probe to understand the properties of dark matter and dark energy [1]. Baryonic acoustic oscillations (BAO) imprinted on the large-scale structure play a role as a standard ruler [2-12] to determine the expansion history of the Universe from various galaxy surveys [13-24]. The overall shape of the matter power spectrum is useful to infer the neutrino mass $[25,26]$. The anisotropy in the redshift-space clustering due to the bulk motion of galaxies provides a key probe to test general relativity [27-33]. One can expect precision cosmological analysis from galaxy clustering in upcoming galaxy surveys such as the Prime Focus Spectrograph [34], the Dark Energy Spectroscopic Instrument [35], the Hobby-Eberly Telescope Dark Energy Experiment [36], Euclid [37], and the Wide Field Infrared Survey Telescope [38].

Nonlinearity in the gravitational evolution of largescale structure makes precise cosmological analysis complicated. The BAO feature is degraded with structure formation mainly due to the bulk motions of matter [39]. The perturbation theory has been derived to describe the

*chiaki.hikage@ipmu.jp nonlinear effects on the power spectra [40-49]; however, the availability is limited to the weakly nonlinear regime even including the higher-order nonlinear terms [50-55]. Evolved density fields no longer follow Gaussian statistics, and thereby their clustering information is not fully described with two-point statistics but leaks to higher-order statistics.

Eisenstein et al. [56] applies a density field reconstruction technique to aim for recovering the original $\mathrm{BAO}$ signature by undoing the bulk motion in the Zeldovich approximation [57]. The method has been extensively studied analytically and tested using numerical simulations [58-62] and applied to the current $\mathrm{BAO}$ analysis $[18-24,63]$. It is also shown that the density field reconstruction recovers the initial density field out to smaller scales using more optimal ways of density field reconstruction beyond the standard reconstruction method [61,64-70].

Although the reconstruction succeeds in the BAO analysis, it is relatively unclear how the reconstructed power spectrum can be described in a perturbative manner. In this paper, we derive the exact one-loop order perturbative formula of the redshift-space matter power spectra after reconstruction. In our previous work, we derive the oneloop perturbative formula of real-space matter power spectra and find that the amplitudes of the one-loop terms decrease significantly and then the perturbation theory can 
be applied to higher wave number $k$. The result is consistent with the previous work showing that the reconstructed field better recovers the initial density field [61]. In this paper, we extend our previous work to redshift-space matter density fields. Recently Chen et al. [71] presented the perturbative formula of halo power spectra in redshift space. Our analysis is limited to the matter power spectra in redshift space, but include the nonlinearities from the Lagrangian to Eulerian mapping in our perturbative formula. From the comparison with large-scale suite of $\mathrm{N}$-body simulations, we study to what extent the monopole and quadrupole spectra of the redshift-space matter fields can be described in one-loop order. We also demonstrate the impact on the cosmic growth rate assuming the survey volume and the number density like the Baryon Oscillation Spectroscopic Survey (BOSS) [23] when modeling the redshift-space power spectra with the one-loop perturbation.

The paper is organized as follows: Sec. II describes the one-loop perturbation theory of the redshift-space matter power spectra and explicitly show the one-loop results in the Appendix. In Sec. III, we study how the one-loop perturbation better describes the redshift-space matter power spectra in comparison with $\mathrm{N}$-body simulations. Section IV is devoted to summary and conclusions.

\section{ONE-LOOP STANDARD PERTURBATION THEORY OF REDSHIFT-SPACE MATTER POWER SPECTRA}

In this section, we derive the perturbative formula based on the standard perturbation theory (SPT) to describe the nonlinearity in the redshift-space matter power spectrum at one-loop order.

The comoving redshift space-position $\mathbf{x}$ is related to the Lagrangian position $\mathbf{q}$ as

$$
\mathbf{x}=\mathbf{q}+\mathbf{\Psi}^{z}(\mathbf{q}),
$$

where $\boldsymbol{\Psi}^{z}$ is the comoving displacement in redshift space given by

$$
\boldsymbol{\Psi}^{z}=\boldsymbol{\Psi}+\frac{\hat{\mathbf{z}} \cdot \dot{\boldsymbol{\Psi}}}{H} \hat{\mathbf{z}}
$$

where $H$ is the time-dependent Hubble parameter and $\hat{\mathbf{z}}$ is the unit vector of the line-of-sight direction. In the Einsteinde-Sitter (EdS) model, the $n$th order perturbative displacement $\Psi^{(\mathrm{n})}$ is proportional to $n$th power of the linear growth factor $D$, and thereby the time derivative of the displacement becomes

$$
\dot{\Psi}^{(\mathrm{n})}=n H f \Psi^{(\mathrm{n})},
$$

where $f=d \ln D / d \ln a$ is the linear growth rate. In a $\Lambda \mathrm{CDM}$ cosmology, the EdS approximation is valid to less than a percent level at the one-loop order of power spectra on the scales of our interest [72-75]. The $n$th order displacement in redshift space then becomes

$$
\Psi^{z(n)}=\mathbf{R}^{(n)} \Psi^{(n)},
$$

where

$$
\mathbf{R}_{i j}^{(n)}=\delta_{i j}+n f \hat{z}_{i} \hat{z}_{j},
$$

and $\delta_{i j}$ is Kronecker delta. The perturbative kernels in redshift space is given by

$$
\mathbf{L}^{z(n)}=\mathbf{R}^{(n)} \mathbf{L}^{(n)} .
$$

The shift field $\mathbf{s}_{z}(\mathbf{x})$ in redshift space is computed from the negative ZA [57] of the smoothed density field as

$$
\begin{aligned}
& \mathbf{s}^{z}(\mathbf{x})=\int \frac{\mathbf{d k}}{(2 \pi)^{3}} \tilde{\mathbf{s}}_{\mathbf{k}}^{z} e^{i \mathbf{k} \cdot \mathbf{x}}, \\
& \tilde{\mathbf{s}}_{\mathbf{k}}^{z}=-i W(k) \mathbf{L}^{(1)}(\mathbf{k}) \tilde{\delta}_{\mathbf{k}}^{z},
\end{aligned}
$$

where $W(k)$ is the smoothing kernel and we adopt a Gaussian kernel $W(k)=\exp \left(-k^{2} R_{s}^{2} / 2\right)$ with the smoothing scale of $R_{s}$. We found that the perturbation works best around $R_{s}=10 h^{-1} \mathrm{Mpc}$ in real space [76], and thereby we fix $R_{s}$ to be $10 h^{-1} \mathrm{Mpc}$ in this paper. The perturbative series of the shift field is given by

$$
\tilde{\mathbf{s}}_{\mathbf{k}}^{z(n)}=-i W(k) \mathbf{L}^{(1)}(\mathbf{k}) \tilde{\delta}_{\mathbf{k}}^{z(n)}
$$

where $\delta_{\mathbf{k}}^{z(n)}$ is the $n$th order perturbation of the redshiftspace density fluctuation. This can be rewritten as

$$
\begin{aligned}
\tilde{\mathbf{S}}_{\mathbf{k}}^{z(n)}= & \frac{i D^{n}}{n !} \int \frac{\mathbf{d k}_{1} \cdots \mathbf{d k}_{n}}{(2 \pi)^{3 n-3}} \delta_{\mathrm{D}}\left(\sum_{j=1}^{n} \mathbf{k}_{j}-\mathbf{k}\right) \\
& \times \mathbf{S}^{z(n)}\left(\mathbf{k}_{1}, \ldots, \mathbf{k}_{n}\right) \tilde{\delta}_{\mathbf{k}_{1}}^{\mathrm{L}} \cdots \tilde{\delta}_{\mathbf{k}_{n}}^{\mathrm{L}},
\end{aligned}
$$

where the kernel of the shift field $\mathbf{S}^{z(n)}$ is written with the redshift-space Eulerian kernel $F_{n}^{z}$ as

$\mathbf{S}^{z(n)}\left(\mathbf{k}_{1}, \ldots, \mathbf{k}_{n}\right)=-n ! W(k) \mathbf{L}^{(1)}(\mathbf{k}) F_{n}^{z}\left(\mathbf{k}_{1}, \ldots, \mathbf{k}_{n}\right)$.

The redshift-space kernel is given in the previous literature $[50,77,78]$,

$$
\begin{gathered}
F_{1}^{z}=1+f \mu^{2}, \\
F_{2}^{z}\left(\mathbf{k}_{1}, \mathbf{k}_{2}\right)=F_{2}\left(\mathbf{k}_{1}, \mathbf{k}_{2}\right)+f \mu^{2} G_{2}\left(\mathbf{k}_{1}, \mathbf{k}_{2}\right) \\
+\frac{1}{2} f k \mu\left(\frac{k_{1 z}}{k_{1}^{2}}+\frac{k_{2 z}}{k_{2}^{2}}\right)+\frac{1}{2}(f k \mu)^{2} \frac{k_{1 z} k_{2 z}}{k_{1}^{2} k_{2}^{2}},
\end{gathered}
$$




$$
\begin{aligned}
F_{3}^{z}\left(\mathbf{k}_{1}, \mathbf{k}_{2}, \mathbf{k}_{3}\right) \\
=F_{3}\left(\mathbf{k}_{1}, \mathbf{k}_{2}, \mathbf{k}_{3}\right)+f \mu^{2} G_{3}\left(\mathbf{k}_{1}, \mathbf{k}_{2}, \mathbf{k}_{3}\right) \\
\quad+f k \mu \frac{k_{1 z}}{k_{1}^{2}} F_{2}\left(\mathbf{k}_{2}, \mathbf{k}_{3}\right)+f k \mu \frac{k_{2 z}+k_{3 z}}{\left|\mathbf{k}_{2}+\mathbf{k}_{3}\right|^{2}} G_{2}\left(\mathbf{k}_{2}, \mathbf{k}_{3}\right) \\
\quad+(f k \mu)^{2} \frac{k_{1 z}\left(k_{2 z}+k_{3 z}\right)}{k_{1}^{2}\left|\mathbf{k}_{2}+\mathbf{k}_{3}\right|^{2}} G_{2}\left(\mathbf{k}_{2}, \mathbf{k}_{3}\right) \\
\quad+\frac{1}{2}(f k \mu)^{2} \frac{k_{1 z} k_{2 z}}{k_{1}^{2} k_{2}^{2}}+\frac{1}{6}(f k \mu)^{3} \frac{k_{1 z} k_{2 z} k_{3 z}}{k_{1}^{2} k_{2}^{2} k_{3}^{2}}
\end{aligned}
$$

where $\mu=\mathbf{k} \cdot \hat{\mathbf{z}} / k$ and $G_{n}$ is the $n$th kernel of peculiar velocity field.

The displaced density field is written as

$$
\tilde{\delta}_{\mathbf{k}}^{\mathrm{z}(\mathrm{d})}=\int \mathbf{d q} e^{-i \mathbf{k} \cdot \mathbf{q}}\left(e^{-i \mathbf{k} \cdot\left[\Psi^{z}(\mathbf{q})+\mathbf{s}^{z}(\mathbf{x})\right]}-1\right),
$$

where the shift field of the evolved mass particles is evaluated at the Eulerian positions $\mathbf{x}$. The difference of the shift field between the Eulerian and Lagrangian positions is perturbatively expanded in terms of $\boldsymbol{\Psi}$ as

$$
\begin{aligned}
\mathbf{s}^{z}(\mathbf{x}) & =\int \frac{\mathbf{d} \mathbf{k}}{(2 \pi)^{3}} \tilde{\mathbf{s}}_{\mathbf{k}}^{z} e^{i \mathbf{k} \cdot\left(\mathbf{q}+\Psi^{\mathbf{z}}(\mathbf{q})\right)}, \\
& =\sum_{n=0}^{\infty} \int \frac{\mathbf{d} \mathbf{k}}{(2 \pi)^{3}} \tilde{\mathbf{s}}_{\mathbf{k}}^{z} e^{i \mathbf{k} \cdot \mathbf{q}}\left[\frac{1}{n !}\left(i \mathbf{k} \cdot \Psi^{\mathbf{z}}(\mathbf{q})\right)^{n}\right], \\
& =\mathbf{s}^{z}(\mathbf{q})+\left(\boldsymbol{\Psi}^{\mathbf{z}}(\mathbf{q}) \cdot \nabla\right) \mathbf{s}^{z}(\mathbf{q})+\frac{1}{2}\left(\Psi^{z}(\mathbf{q}) \cdot \nabla\right)^{2} \mathbf{s}^{z}(\mathbf{q}) \cdots
\end{aligned}
$$

The shifted density field of a spatially uniform grid or random is given by

$$
\tilde{\delta}_{\mathbf{k}}^{z(s)}=\int \mathbf{d q} e^{-i \mathbf{k} \cdot \mathbf{q}}\left(e^{-i \mathbf{k} \cdot \mathbf{s}^{z}(\mathbf{q})}-1\right),
$$

where the shift field of the (unevolved) uniform grid is evaluated at the Lagrangian position. The reconstructed density field in redshift space is given as

$$
\begin{aligned}
\tilde{\delta}_{\mathbf{k}}^{z(\mathrm{rec})} & \equiv \tilde{\delta}_{\mathbf{k}}^{z(\mathrm{~d})}-\tilde{\delta}_{\mathbf{k}}^{z(\mathrm{~s})} \\
& =\int \mathbf{d q} e^{-i \mathbf{k} \cdot \mathbf{q}} e^{-i \mathbf{k} \cdot \mathbf{s}^{z}(\mathbf{q})}\left(e^{-i \mathbf{k} \cdot\left[\Psi^{z}(\mathbf{q})+\mathbf{s}^{z}(\mathbf{x})-\mathbf{s}^{z}(\mathbf{q})\right]}-1\right)
\end{aligned}
$$

The redshift-space formula is the same as that in real space but replacing the real-space kernels $\mathbf{L}^{(n)}$ and $F_{n}$ with the redshift-space ones $\mathbf{L}^{z(n)}$ and $F_{n}^{z}$.

At linear order, the reconstructed density field in redshift space is not changed by reconstruction

$$
\delta_{\mathbf{k}}^{z(\operatorname{rec})(1)}=\delta_{\mathbf{k}}^{z(1)} .
$$

Higher-order terms of $\delta^{(\mathrm{rec})}$ are given by

$$
\begin{aligned}
\tilde{\delta}_{\mathbf{k}}^{\mathrm{z}(\mathrm{rec}, \mathrm{n})}= & D^{n}(z) \int \frac{\mathbf{d k}_{1} \cdots \mathbf{d} \mathbf{k}_{n}}{(2 \pi)^{3 n-3}} \delta_{\mathrm{D}}\left(\sum_{j=1}^{n} \mathbf{k}_{j}-\mathbf{k}\right) \\
& \times F_{n}^{\mathrm{z}(\mathrm{rec})}\left(\mathbf{k}_{1}, \ldots, \mathbf{k}_{n}\right) \tilde{\delta}_{\mathbf{k}_{1}}^{\mathrm{L}} \cdots \tilde{\delta}_{\mathbf{k}_{n}}^{\mathrm{L}},
\end{aligned}
$$

where $F_{n}^{z(\mathrm{rec})}$ is the Eulerian kernel for the reconstructed matter density field in redshift space. We have already derived the explicit form of the reconstructed Eulerian kernel in real space in the previous paper [76]. The firstorder Eulerian kernel does not change after reconstruction,

$$
F_{1}^{\mathrm{z}(\mathrm{rec})}=F_{1}^{\mathrm{z}} .
$$

The second-order Eulerian kernel for the reconstructed field $F_{2}^{z(\text { rec })}$ can be derived by replacing the real-space kernel to the redshift-space one in the equation of (32) or (A10) in Hikage et al. [76] as

$$
\begin{aligned}
F_{2}^{\mathrm{z}(\mathrm{rec})}\left(\mathbf{k}_{1}, \mathbf{k}_{2}\right)= & F_{2}^{z}\left(\mathbf{k}_{1}, \mathbf{k}_{2}\right) \\
& +\frac{1}{2}\left[\left(\mathbf{k} \cdot \mathbf{S}^{\mathrm{z}(1)}\left(\mathbf{k}_{1}\right)\right)\left(\mathbf{k}_{2} \cdot \mathbf{L}^{z(1)}\left(\mathbf{k}_{2}\right)\right)\right. \\
& \left.+\left(\mathbf{k} \cdot \mathbf{S}^{\mathrm{z}(1)}\left(\mathbf{k}_{2}\right)\right)\left(\mathbf{k}_{1} \cdot \mathbf{L}^{z(1)}\left(\mathbf{k}_{1}\right)\right)\right] .
\end{aligned}
$$

Note that $\mathbf{k}_{i} \cdot \mathbf{L}^{(1)}\left(\mathbf{k}_{i}\right)$ terms in the real space becomes unity and thereby they are not written explicitly in the realspace formula. In redshift space, however, $\mathbf{k}_{i} \cdot \mathbf{L}^{(1)}\left(\mathbf{k}_{i}\right)$ becomes $1+f \mu_{i}^{2}$ where $\mu_{i}=\mathbf{k}_{\mathbf{i}} \cdot \hat{\mathbf{z}}$ and thus the $F_{2}^{z(\mathrm{rec})}\left(\mathbf{k}_{1}, \mathbf{k}_{2}\right)$ depends on the line-of-sight direction of the two wave numbers $\mathbf{k}_{1}$ and $\mathbf{k}_{2}$. This makes the one-loop perturbative formula complicated as shown in Appendix.

The third-order kernel is also derived by replacing the real-space kernel with the redshift space one in the equation of (33) or (A29) in [76] as

$$
\begin{aligned}
F_{3}^{z(\mathrm{rec})}\left(\mathbf{k}_{1}, \mathbf{k}_{2}, \mathbf{k}_{3}\right) \\
=F_{3}^{z}\left(\mathbf{k}_{1}, \mathbf{k}_{2}, \mathbf{k}_{3}\right)+\frac{1}{6}\left[2\left(\mathbf{k} \cdot \mathbf{S}^{z(1)}\left(\mathbf{k}_{1}\right)\right) F_{2}^{z}\left(\mathbf{k}_{2}, \mathbf{k}_{3}\right)\right. \\
\quad+\left(\mathbf{k} \cdot \mathbf{S}^{z(1)}\left(\mathbf{k}_{1}\right)\right)\left(\mathbf{k} \cdot \mathbf{S}^{z(1)}\left(\mathbf{k}_{2}\right)\right)\left(\mathbf{k}_{3} \cdot \mathbf{L}^{z(1)}\left(\mathbf{k}_{3}\right)\right) \\
\quad+\left(\mathbf{k} \cdot \mathbf{S}^{z(2)}\left(\mathbf{k}_{1}, \mathbf{k}_{2}\right)\right)\left(\mathbf{k}_{3} \cdot \mathbf{L}^{z(1)}\left(\mathbf{k}_{3}\right)\right) \\
\quad+(2 \text { perms. })] .
\end{aligned}
$$

The third-order kernel also depends on the line-of-sight direction of three wave numbers $\mu_{i}$ with $i=1,2$, and 3 .

The reconstructed power spectrum at one-loop order is written by 
$P^{\mathrm{z}(\mathrm{rec}), 1-\operatorname{loop}}(k)=D^{2}(z) P_{11}^{\mathrm{z}(\mathrm{rec})}(k)+D^{4}(z)\left(P_{22}^{\mathrm{z}(\mathrm{rec})}+P_{13}^{\mathrm{z}(\mathrm{rec})}\right)$,

where $P_{n m}^{z(\mathrm{rec})}=\left\langle\tilde{\delta}_{\mathbf{k}}^{(\mathrm{n})} \delta_{\mathbf{k}}^{(\mathrm{m})}\right\rangle$. The leading-order term is unchanged after reconstruction,

$$
P_{11}^{\mathrm{z}(\mathrm{rec})}(k, \mu)=\left(1+f \mu^{2}\right)^{2} P_{\mathrm{L}}(k) .
$$

The one-loop terms of the redshift-space power spectrum can be written with the reconstructed Eulerian kernels as

$$
\begin{aligned}
P_{22}^{\mathrm{z}(\mathrm{rec})}(k, \mu)= & 2 \int \frac{\mathbf{d p}}{(2 \pi)^{3}} P_{\mathrm{L}}(|\mathbf{k}-\mathbf{p}|) P_{\mathrm{L}}(p) \\
& \times\left[F_{2}^{\mathrm{z}(\mathrm{rec})}(\mathbf{k}-\mathbf{p}, \mathbf{p})\right]^{2}
\end{aligned}
$$

and

$$
\begin{aligned}
P_{13}^{\mathrm{z}(\mathrm{rec})}(k, \mu)= & 6 F_{1}^{\mathrm{z}(\mathrm{rec})}(k) P_{\mathrm{L}}(k) \\
& \times \int \frac{\mathbf{d p}}{(2 \pi)^{3}} P_{\mathrm{L}}(p) F_{3}^{\mathrm{z}(\mathrm{rec})}(\mathbf{k}, \mathbf{p},-\mathbf{p}) .
\end{aligned}
$$

The exact formula of the one-loop terms is summarized in Appendix. The multipole components of the redshift-space power spectrum is generally obtained by the Legendre polynomial expansion as

$$
P_{\ell}(k)=\frac{1}{2} \int_{-1}^{1} d \mu P(k, \mu) \mathcal{L}_{\ell}(\mu),
$$

where $\mathcal{L}_{\ell}(\mu)$ is the Legendre polynomials, for example, $\mathcal{L}_{0}(\mu)=1$ and $\mathcal{L}_{2}(\mu)=\left(3 \mu^{2}-1\right) / 2$.

Figure 1 shows the $k$ dependence of the one-loop terms $P_{13}$ and $P_{22}$ in the monopole $(\ell=0)$ and quadrupole $(\ell=2)$ spectra before and after reconstruction. We find that the amplitudes of both one-loop terms significantly decrease after reconstruction in monopole and quadrupole spectra out to large $k$. This result is similar to the results in real space [cf. Fig. 1 of [76]], but indicates that mode couplings between density and velocity fields due to nonlinear gravity are partially removed by reconstruction. In-phase baryonic acoustic oscillations of $P_{13}$, but with negative amplitude, cause the degradation of the BAO signature. The oscillation of $P_{13}$ also significantly reduces after reconstruction and thereby the original $\mathrm{BAO}$ signature is substantially recovered. The result is consistent with that the BAO feature in redshift-space spectra is actually recovered by the reconstruction [63].

\section{RESULTS}

We compute the redshift-space matter power spectra using $N$-body simulations to see how well the one-loop perturbative formula describes the reconstructed spectra. Dark-matter $N$-body simulations are performed using a

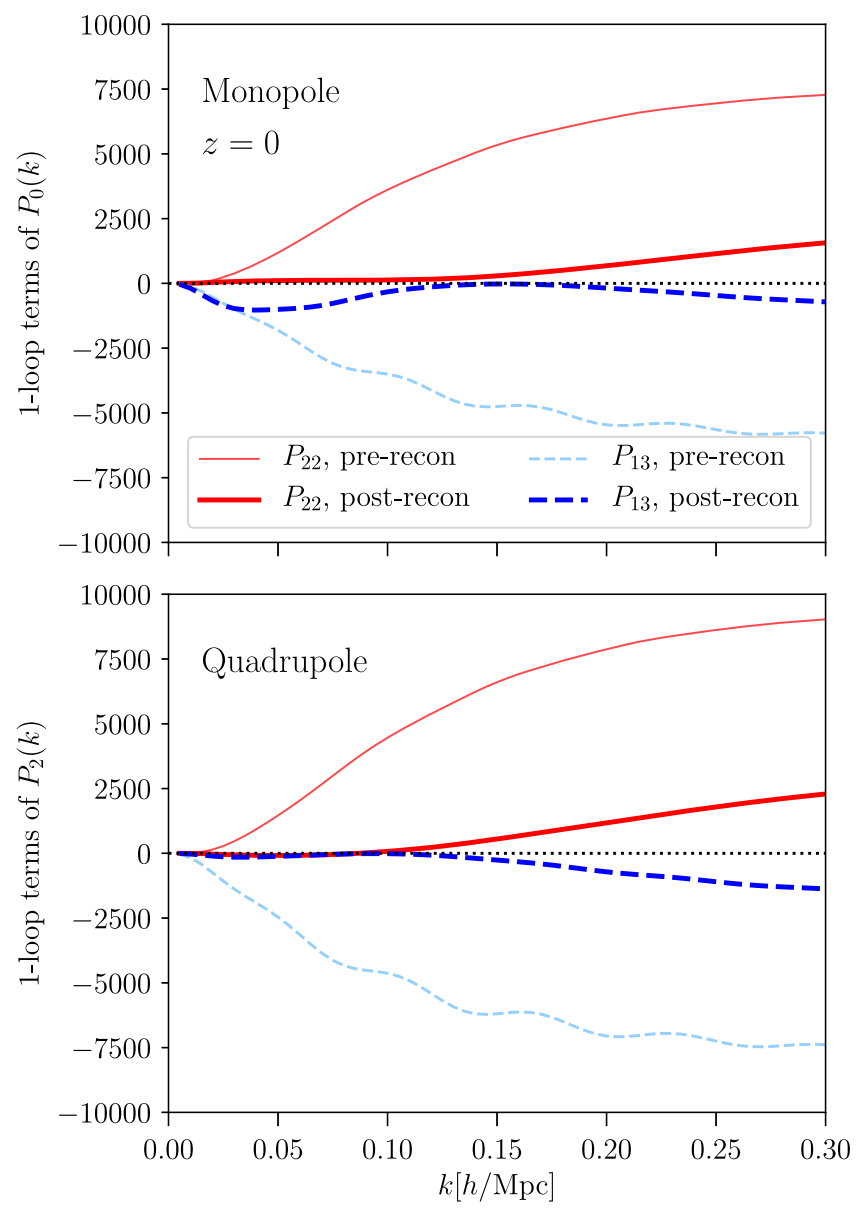

FIG. 1. Comparison of the one-loop terms $P_{22}(k)$ and $P_{13}(k)$ in monopole (upper) and quadrupole (lower) spectra of redshiftspace matter density fields before (thin) and after (thick) reconstruction. The amplitudes of the both one-loop terms significantly decrease by reconstruction out to large $k$.

publicly available code Gadget-2 [79]. The mass particles are initially distributed based on 2LPT code [80,81] with Gaussian initial conditions at the input redshift of 31. The initial linear power spectrum is computed by CAMB [82]. Each simulation is performed in a cubic box with the side length of $4 h^{-1}$ Gpc with $4096^{3}$ particles. We assign the $N$-body particles to $2048^{3}$ grid cells to calculate the density contrast and then perform the Fourier transform [83] to measure the power spectrum. In our analysis, we use eight realizations with two output redshifts of $z=0$ and $z=1.02$. We will show the average power spectrum with $1 \sigma$ error estimated from these realizations. The cosmology in the simulations is based on a flat $\Lambda \mathrm{CDM}$ model with the best-fit values of Planck TT,TE,EE+lowP in 2015, i.e., $\Omega_{b}=0.0492, \Omega_{m}=0.3156, h=0.6727, n_{s}=0.9645$, and $\sigma_{8}=0.831$ [84].

We evaluate the agreement between the simulated power spectra and the perturbative formula with the following $\chi^{2}$ value: 

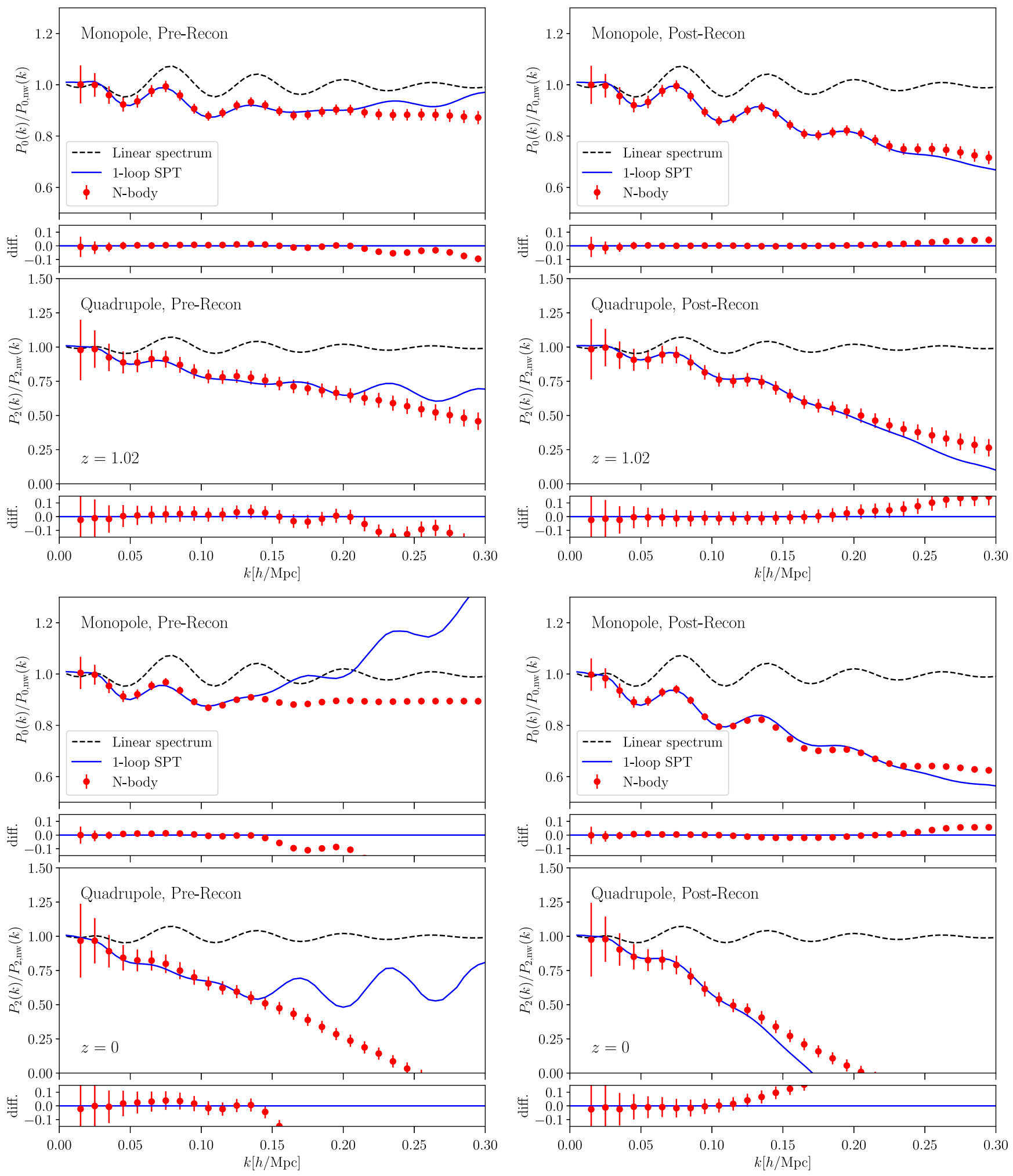

FIG. 2. Monopoles and quadrupoles of simulated redshift-space matter power spectrum (filled circles) before (left) and after (right) reconstruction at $z=1.02$ (upper) and $z=0$ (lower). For comparison, we plot the one-loop SPT (solid lines) with two counterterms proportional to $P_{\ell}^{(\text {lin) }}(k) k^{2}$ fitted to the simulated spectra up to $k=0.2 h / \mathrm{Mpc}$ for $z=1.02$ and $k=0.12 h / \mathrm{Mpc}$ for $z=0$. For references, the linear spectra are plotted with lines. All of the power spectra are normalized with the no-wiggle multipole spectra in redshift space. The plotted error bars are Gaussian error assuming a BOSS-like survey with the survey volume $V=6\left(h^{-1} \mathrm{Gpc}\right)^{3}$ and the number density $n=2 \times 10^{-4}\left(h^{-1} \mathrm{Mpc}\right)^{-3}$. Difference ratios between simulated spectra and one-loop spectra are also shown under each panel. The figure shows that their agreement becomes better after reconstruction and the perturbation works at higher $k$. 


$$
\begin{aligned}
\chi^{2}= & \sum_{i}^{k_{\min } \leq k_{i} \leq k_{\max }} \sum_{\ell \ell^{\prime}}^{0,2}\left[P_{\ell}^{\text {theory }}\left(k_{i}\right)-P_{\ell}^{\operatorname{sim}}\left(k_{i}\right)\right] \\
& \times \operatorname{Cov}_{\ell \ell^{\prime}}^{-1}\left(k_{i}\right)\left[P_{\ell^{\prime}}^{\text {theory }}\left(k_{i}\right)-P_{\ell^{\prime}}^{\operatorname{sim}}\left(k_{i}\right)\right],
\end{aligned}
$$

where $\operatorname{Cov}_{\ell \ell^{\prime}}\left(k_{i}\right)$ represents the covariance of multipole power spectra at a given $k_{i}$. Here, we focus on the monopole and quadrupole components of redshift-space matter power spectra. For simplicity, we adopt the analytical formula of the Gaussian covariance given by the Appendix C of Taruya et al. [52] with typos corrected and neglect the off-diagonal components of the covariance between different bins of $k$. This approximation would be valid when the cosmic variance and/or shot-noise terms are dominant compared to the non-Gaussian terms. The Gaussian covariance depends on the survey volume $V$ and the number density $n$. In this paper, we assume BOSS-like survey with $V=6\left(h^{-1} \mathrm{Gpc}\right)^{3}$ and $n=2 \times 10^{-4}\left(h^{-1} \mathrm{Mpc}\right)^{-3}$. The chi-squared value depends on the range of $k$. Here we fix the minimum value $k_{\min }=$ $0.01 \mathrm{~h} \mathrm{Mpc}^{-1}$ and see how $\chi^{2}$ changes as the maximum value $k_{\max }$ increases. For the theoretical power spectrum $P^{\text {theory }}(k)$, we adopt the one-loop perturbative formula with the lowest-order counter term proportional to $k^{2} P_{\ell}$ in each $\ell$,

$$
P_{\ell}^{\text {theory }}(k)=P_{\ell}^{\mathrm{z}, 1-\text { loop }}(k)+\alpha_{\ell} k^{2} P_{\ell}^{\mathrm{L}}(k) .
$$

The counterterms renormalize the contributions from UV (small-scale) power $[85,86]$ ncluding the lowest-order contributions of nonlinear redshift-space distortions, i.e., fingers-of-God effect. The proportional factor $\alpha_{\ell}(\ell=0$ and 2) is obtained by fitting them to the simulated power spectra. Note that we adopt one counterterm per each multipole for both prerecon and postrecon spectrum, while [71] adopts three counterterms per each multipole proportional to the power spectra for $\left\langle\tilde{\delta}_{k}^{z(d)} \tilde{\delta}_{k}^{* z(d)}\right\rangle,\left\langle\tilde{\delta}_{k}^{z(d)} \tilde{\delta}_{k}^{* z(s)}\right\rangle$, $\left\langle\tilde{\delta}_{k}^{z(s)} \tilde{\delta}_{k}^{* z(s)}\right\rangle$ for the reconstructed spectrum.

Figure 2 shows the comparison of the monopole and quadrupole of simulated power spectrum with the one-loop perturbative formulae before (left) and after reconstruction (right) at the output redshift of 1.02 (upper) and 0 (lower). We adopt the best-fit values (the minimum $\chi^{2}$ ) of counterterms with $k_{\max }$ of $0.2 h / \mathrm{Mpc}$ for $z=1$ and $0.12 h / \mathrm{Mpc}$ for $z=0$ where the minimum $\chi^{2}$ value is less than unity. In these plots, the power spectra are normalized with redshift-space no-wiggle spectrum including linear Kaiser effect [87], i.e., $P^{\mathrm{nw}, \mathrm{z}}(k, \mu)=\left(1+f \mu^{2}\right)^{2} P^{\mathrm{nw}}(k)$ where $f \equiv$ $d \ln D(z) / d \ln a$ is the growth rate at a given $z$ and $P^{\text {nw }}(k)$ is the no-wiggle spectrum given by Eisenstein and $\mathrm{Hu}$ [88]. We find that the one-loop perturbative formula can be better fitted to the postrecon spectrum up to higher $k$. More quantitatively saying, $k_{\max }$ where $\chi_{\min }^{2}$ becomes unity is $0.17 \mathrm{~h} / \mathrm{Mpc}$ for $z=1$ and $0.11 \mathrm{~h} / \mathrm{Mpc}$ for $z=0$ before
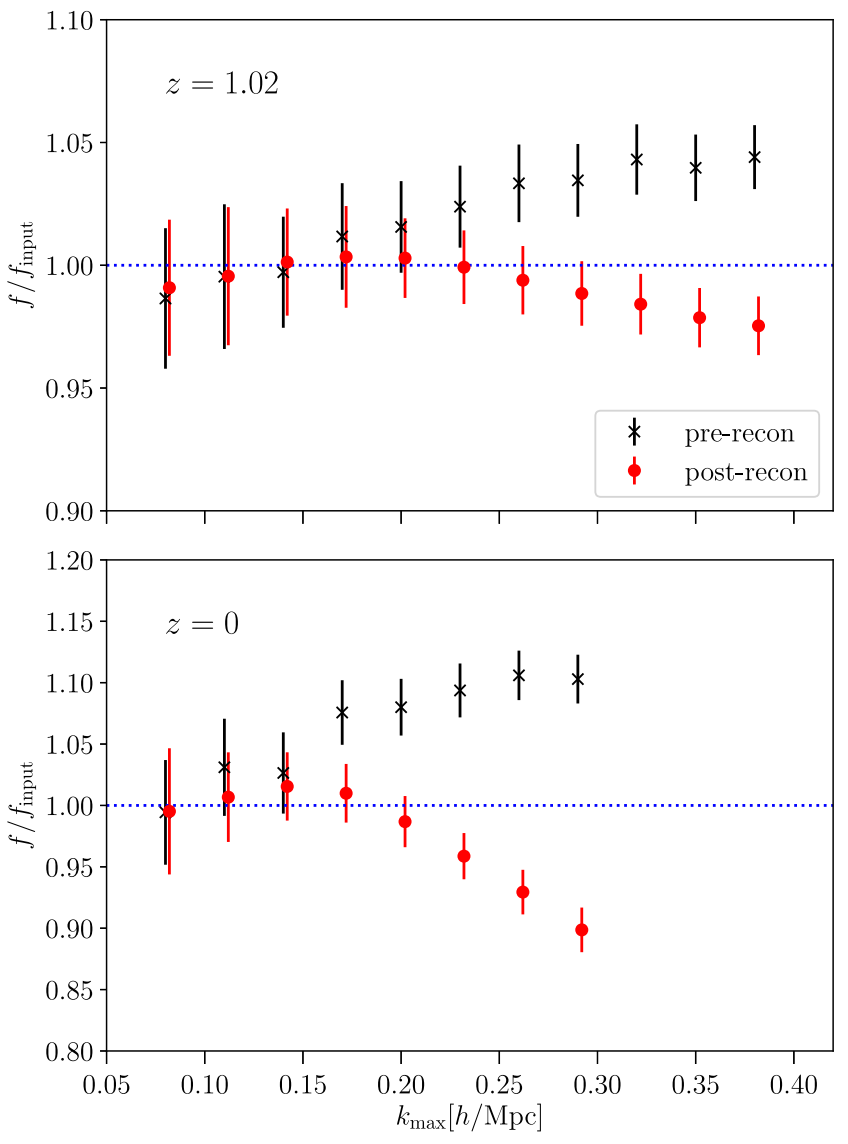

FIG. 3. Comparison of the $1 \sigma$ statistical error and the systematic bias on the growth rate $f$ expected from the monopole and quadrupole matter power spectra as a function of $k_{\max }$ before and after reconstruction. We again assume a BOSS-like survey volume and number density, i.e., $V=6\left(h^{-1} \mathrm{Gpc}\right)^{3}$ and $n=2 \times 10^{-4}\left(h^{-1} \mathrm{Mpc}\right)^{-3}$, to compute the error of the multipole power spectra, but the output redshift is $z=1.02$ (upper) and $z=0$ (lower), respectively. The one-loop perturbation theory is used to fit the simulated matter power spectra and thereby the systematic error becomes significant at higher $k_{\max }$. The figure shows that the reconstructed spectra better reproduce the input value of $f$ and then the systematic error exceeds the statistical error at higher $k_{\max }$ by reconstruction.

reconstruction, which are extended to be $0.23 h / \mathrm{Mpc}$ for $z=1$ and $0.13 h / \mathrm{Mpc}$ for $z=0$ after reconstruction.

We also see the impact on the measurement of the growth rate $f$ by computing the likelihood function $\mathcal{L} \propto$ $\exp \left(-\chi^{2} / 2\right)$ where $\chi^{2}$ is computed from Eq. (30). In addition to the counterterms $\alpha_{0}$ and $\alpha_{2}$, the growth rate $f$ is treated as free parameters. Figure 3 shows the expected constraints on $f$ from the monopole and quadrupole spectra with different $k_{\max }$. Here we again assume the error expected from the same BOSS-like survey volume and number density. The statistical error decreases at higher $k_{\max }$; however, the systematic error increases because the one-loop approximation becomes worse at higher $k$. We find that $k_{\max }$ where the statistical error is comparable to the 
systematic one is $0.22 h / \mathrm{Mpc}$ for $z=1$ and $0.12 h / \mathrm{Mpc}$ for $z=0$ before reconstruction. The corresponding wave numbers are extended to be $0.30 \mathrm{~h} / \mathrm{Mpc}$ for $z=1$ and $0.21 \mathrm{~h} / \mathrm{Mpc}$ for $z=0$ after reconstruction. The errors at the $k_{\max }$ where the statistical error is comparable to the systematic one decreases from 0.0171 to 0.0128 (40\% decrement) for $z=1$ and from 0.0405 to 0.0197 ( $51 \%$ decrement) for $z=0$ by reconstruction.

\section{SUMMARY AND CONCLUSIONS}

We derived the one-loop perturbative formulae of the redshift-space matter power spectra after density-field reconstruction using the Zeldovich approximation. We found that the amplitudes of the one-loop nonlinear terms $P_{13}(k)$ and $P_{22}(k)$ decrease significantly in both monopole and quadrupole spectra. Our result indicates that the mode couplings among density and velocity fields associated with nonlinear gravity are partly eliminated by the reconstruction. From the comparison of $N$-body simulations, we showed that the one-loop perturbative formulae better describe the monopole and quadrupole of matter power spectra after reconstruction and agree with the simulated spectra at higher $k$. We also estimated the impact on the measurement of the growth rate when using the oneloop perturbation theory as a theoretical modeling of the redshift-space matter power spectra assuming the survey volume and number density of a BOSS-like galaxy survey. We found that the systematics due to the one-loop approximation is reduced by reconstruction and thereby the total error of the growth rate measurement including the statistical and systematic errors decreases by half.

In this paper, we focused on the redshift-space matter power spectra. We plan to extend our analysis to the power spectra of biased tracers but leave this work in the near future. In this analysis, we neglected the non-Gaussianity in the covariance of matter power spectra. Since the leadingorder non-Gaussianity also comes from the one-loop terms [89,90], the non-Gaussianity should be smaller after reconstruction and thereby the information content of the power spectrum is expected to increase by reconstruction. We plan to show more detailed analysis of the covariance of reconstructed power spectra in the near future.

\section{ACKNOWLEDGMENTS}

We thank Alan Heavens and Marcel Schmittfull for useful discussion. This work is supported by MEXT/JSPS
KAKENHI Grants No. JP16K17684 (C. H.), No. JP18H04348 (C. H.), No. JP15H05893 (R. T.), and No. JP17H01131 (R. T.). K. K. is supported by the UK STFC Grants No. ST/N000668/1 and No. ST/S000550/1, and the European Research Council under the European Union's Horizon 2020 programme (Grant No. 646702 "CosTesGrav"). Numerical computations were in part carried out on Cray XC30 and XC50 at Centre for Computational Astrophysics, National Astronomical Observatory of Japan.

\section{APPENDIX: DERIVATION OF THE ONE-LOOP MATTER POWER SPECTRUM IN REDSHIFT SPACE}

The one-loop terms of the redshift-space matter power spectra $P_{13}(k)$ and $P_{22}(k)$ are derived from Eqs. (27) and (28) after a lengthy but straightforward calculation. Their equations are summarized below after the integration over the azimuthal angle of $\mathbf{p}$ as follows:

$$
\begin{aligned}
P_{22}^{\mathrm{z}(\mathrm{rec})}(\mathbf{k})= & \sum_{n, m} \mu^{2 n} f^{m} \frac{k^{3}}{4 \pi^{2}} \int_{0}^{\infty} d r P_{\mathrm{L}}(k r) \\
& \times \int_{-1}^{1} d x P_{\mathrm{L}}\left(k\left(1+r^{2}-2 r x\right)^{1 / 2}\right) \frac{A_{n m}(r, x)}{\left(1+r^{2}-2 r x\right)^{2}}
\end{aligned}
$$

and

$$
\begin{aligned}
P_{13}^{\mathrm{z}(\mathrm{rec})}(\mathbf{k})= & \left(1+f \mu^{2}\right) P_{\mathrm{L}}(k) \sum_{n, m} \mu^{2 n} f^{m} \frac{k^{3}}{4 \pi^{2}} \int_{0}^{\infty} d r P_{0}(k r) \\
& \times \int_{-1}^{1} d x B_{n m}(r, x),
\end{aligned}
$$

where $A_{n m}$ and $B_{n m}$ are the coefficients of $\mu^{2 n} f^{m}$ terms in the one-loop terms. The reconstructed spectra depend on the smoothing kernel in Eq. (8), which are used to derive the shift field from the smoothed density field, and thereby the following equations of the coefficients of the one-loop terms include $W(|\mathbf{p}|)$ and $W(|\mathbf{k}-\mathbf{p}|)$, which are denoted as $W_{p}$ and $W_{\star}$, respectively. These equations agree with the SPT calculation [50] at the limit of prereconstruction, i.e., $W_{p} \rightarrow 0$ and $W_{\star} \rightarrow 0$. The nonvanishing components of $A_{n m}$ and $B_{n m}$ are summarized below.

$$
\begin{gathered}
A_{00}=\frac{\left(r\left(7 r x\left(W_{\star}-W_{p}\right)+2\left(7 W_{p}-5\right) x^{2}-7 W_{\star}+3\right)-7\left(W_{p}-1\right) x\right)^{2}}{98}, \\
A_{01}=-\frac{1}{14\left(r^{2}-2 r x+1\right)}\left[\left(x^{2}-1\right)(2 r(r-x)+1)\left(r\left(W_{p} x(r-2 x)-r W_{\star} x+W_{\star}\right)+W_{p} x\right)\right. \\
\left.\times\left(r\left(7 r x\left(W_{p}-W_{\star}\right)+2\left(5-7 W_{p}\right) x^{2}+7 W_{\star}-3\right)+7\left(W_{p}-1\right) x\right)\right],
\end{gathered}
$$




$$
\begin{aligned}
& A_{11}=\frac{1}{98\left(r^{2}-2 r x+1\right)}\left[\left(r\left(7 r x\left(W_{\star}-W_{p}\right)+2\left(7 W_{p}-5\right) x^{2}-7 W_{\star}+3\right)-7\left(W_{p}-1\right) x\right)\right. \\
& \times\left(-14 r^{4} x\left(3 x^{2}-1\right)\left(W_{p}-W_{\star}\right)+2 r^{3}\left(-21 x^{4}\left(W_{\star}-3 W_{p}\right)-x^{2}\left(7 W_{p}+28 W_{\star}+20\right)+7 W_{\star}+6\right)\right. \\
& +r^{2} x\left(x^{2}\left(-91 W_{p}+63 W_{\star}+80\right)-84 W_{p} x^{4}+7 W_{p}+21 W_{\star}+4\right) \\
& \left.\left.+r\left(x^{2}\left(28 W_{p}-21 W_{\star}-96\right)+84 W_{p} x^{4}-7 W_{\star}+12\right)-7 x\left(3 W_{p} x^{2}+W_{p}-4\right)\right)\right] \text {, } \\
& A_{02}=\frac{3\left(x^{2}-1\right)^{2}}{112\left(r^{2}-2 r x+1\right)^{2}}\left[\left(2 r^{2}\left(r^{2}-2 r x+1\right)\left(-r\left(W_{p} x(r-2 x)-r W_{\star} x+W_{\star}\right)-W_{p} x\right)\right.\right. \\
& \times\left(r\left(7 r x\left(W_{\star}-W_{p}\right)+2\left(7 W_{p}-5\right) x^{2}-7 W_{\star}+3\right)-7\left(W_{p}-1\right) x\right) \\
& \left.\left.+7(2 r(r-x)+1)^{2}\left(r\left(W_{p} x(r-2 x)-r W_{\star} x+W_{\star}\right)+W_{p} x\right)^{2}\right)\right] \text {, } \\
& A_{12}=-\frac{1}{56\left(r^{2}-2 r x+1\right)^{2}}\left[( x ^ { 2 } - 1 ) \left(126 r^{8} x^{2}\left(5 x^{2}-1\right)\left(W_{p}-W_{\star}\right)^{2}\right.\right. \\
& +2 r^{7} x\left(W_{p}-W_{\star}\right)\left(30 x^{4}\left(-63 W_{p}+21 W_{\star}+5\right)+9 x^{2}\left(14 W_{p}+84 W_{\star}+13\right)-126 W_{\star}-1\right) \\
& +2 r^{6}\left(30 x^{6}\left(133 W_{p}^{2}-2 W_{p}\left(49 W_{\star}+10\right)+W_{\star}\left(7 W_{\star}+10\right)\right)\right. \\
& +3 x^{4}\left(497 W_{p}^{2}-W_{p}\left(1624 W_{\star}+295\right)+89 W_{\star}\left(7 W_{\star}+3\right)\right) \\
& \left.+x^{2}\left(112 W_{p} W_{\star}-W_{p}\left(119 W_{p}+111\right)+574 W_{\star}^{2}+230 W_{\star}+48\right)-63 W_{\star}^{2}-W_{\star}-6\right)+4 r^{5} x\left(-105 W_{p}^{2}\right. \\
& \times\left(16 x^{6}+27 x^{4}+x^{2}\right)+W_{p}\left(60\left(7 W_{\star}+5\right) x^{6}+42\left(84 W_{\star}+29\right) x^{4}+\left(1498 W_{\star}+501\right) x^{2}-56 W_{\star}+11\right) \\
& \left.-W_{\star}\left(35 W_{\star}\left(9 x^{4}+29 x^{2}+2\right)+642 x^{4}+655 x^{2}+68\right)-96 x^{2}-9\right) \\
& +r^{4}\left(x^{4}\left(5691 W_{p}^{2}-14 W_{p}\left(881 W_{\star}+430\right)+15 W_{\star}\left(91 W_{\star}+270\right)+384\right)\right. \\
& +x^{2}\left(-63 W_{p}^{2}-14 W_{p}\left(99 W_{\star}+56\right)+W_{\star}\left(1981 W_{\star}+1784\right)+480\right) \\
& \left.+1680 W_{p}^{2} x^{8}+168 W_{p} x^{6}\left(74 W_{p}-25 W_{\star}-27\right)+2\left(W_{\star}\left(7 W_{\star}+23\right)-5\right)\right) \\
& +2 r^{3} x\left(-28 W_{p}^{2} x^{2}\left(60 x^{4}+158 x^{2}+21\right)+W_{p}\left(42\left(45 W_{\star}+73\right) x^{4}+\left(2485 W_{\star}+1577\right) x^{2}+49 W_{\star}+47\right)\right. \\
& \left.-217 W_{\star}^{2}-\left(W_{\star}\left(315 W_{\star}+1493\right)+360\right) x^{2}-257 W_{\star}-88\right) \\
& +r^{2}\left(x^{2}\left(84 W_{p}^{2}-2 W_{p}\left(469 W_{\star}+351\right)+3 W_{\star}\left(35 W_{\star}+348\right)+488\right)\right. \\
& \left.+2520 W_{p}^{2} x^{6}+2 W_{p} x^{4}\left(1512 W_{p}-735 W_{\star}-1945\right)+35 W_{\star}^{2}+48 W_{\star}+16\right) \\
& +2 r x\left(W_{p}\left(x^{2}\left(-252 W_{p}+105 W_{\star}+592\right)-420 W_{p} x^{4}+35 W_{\star}+24\right)-70\left(W_{\star}+1\right)\right) \\
& \left.\left.+35 W_{p} x^{2}\left(3 W_{p} x^{2}+W_{p}-4\right)+14\right)\right] \text {, } \\
& A_{22}=\frac{1}{784\left(r^{2}-2 r x+1\right)^{2}}\left[294 r^{8} x^{2}\left(35 x^{4}-30 x^{2}+3\right)\left(W_{p}-W_{\star}\right)^{2}\right. \\
& +14 r^{7} x\left(W_{p}-W_{\star}\right)\left(70 x^{6}\left(-63 W_{p}+21 W_{\star}+5\right)+x^{4}\left(2940 W_{p}+1050 W_{\star}+299\right)\right. \\
& \left.+18 x^{2}\left(7 W_{p}-91 W_{\star}-18\right)+126 W_{\star}+11\right)+2 r^{6}\left(490 x^{8}\left(133 W_{p}^{2}-2 W_{p}\left(49 W_{\star}+10\right)+W_{\star}\left(7 W_{\star}+10\right)\right)\right. \\
& +x^{4}\left(-23520 W_{p}^{2}+14 W_{p}\left(3906 W_{\star}+673\right)-147 W_{\star}\left(45 W_{\star}+19\right)+2832\right) \\
& +x^{2}\left(343 W_{p}^{2}+133 W_{p}\left(28 W_{\star}+13\right)-7 W_{\star}\left(1463 W_{\star}+593\right)-1884\right) \\
& \left.-7 x^{6}\left(\left(8540 W_{p}-2297\right) W_{\star}+5 W_{p}\left(259 W_{p}+509\right)-4375 W_{\star}^{2}\right)+441 W_{\star}^{2}+77 W_{\star}+228\right) \\
& +4 r^{5} x\left(-7 x^{6}\left(5215 W_{p}^{2}-2 W_{p}\left(4130 W_{\star}+1823\right)+W_{\star}\left(735 W_{\star}+1874\right)\right)\right. \\
& +x^{4}\left(27146 W_{p}^{2}+7 W_{p}\left(151-294 W_{\star}\right)-49 W_{\star}\left(344 W_{\star}+239\right)-5664\right) \\
& +x^{2}\left(3871 W_{p}^{2}-28 W_{p}\left(763 W_{\star}+233\right)+7 W_{\star}\left(1197 W_{\star}+850\right)+1878\right) \\
& \left.-980 W_{p} x^{8}\left(28 W_{p}-7 W_{\star}-5\right)-98 W_{p} W_{\star}-259 W_{p}+1862 W_{\star}^{2}+1239 W_{\star}+258\right)
\end{aligned}
$$




$$
\begin{aligned}
& +r^{4}\left(x^{6}\left(8281 W_{p}^{2}-14 W_{p}\left(14679 W_{\star}+8494\right)+49 W_{\star}\left(455 W_{\star}+1942\right)+22656\right)\right. \\
& +2 x^{4}\left(-36701 W_{p}^{2}+14 W_{p}\left(2989 W_{\star}+1318\right)+35 W_{\star}\left(490 W_{\star}+293\right)+13248\right) \\
& -7 x^{2}\left(217 W_{p}^{2}-2 W_{p}\left(1841 W_{\star}+894\right)+W_{\star}\left(2891 W_{\star}+2914\right)+2084\right) \\
& \left.+27440 W_{p}^{2} x^{10}+56 W_{p} x^{8}\left(3640 W_{p}-1225 W_{\star}-1699\right)-14 W_{\star}\left(77 W_{\star}+85\right)+716\right) \\
& +2 r^{3} x\left(-392 W_{p}^{2}\left(70 x^{6}+189 x^{4}-67 x^{2}-24\right) x^{2}\right. \\
& +7 W_{p}\left(2\left(2205 W_{\star}+5273\right) x^{6}+\left(6223 W_{\star}+3347\right) x^{4}-4\left(847 W_{\star}+488\right) x^{2}-189 W_{\star}-181\right) \\
& -3\left(245 W_{\star}\left(7 W_{\star}+53\right)+8816\right) x^{4}-14 W_{\star}\left(301 W_{\star}+13\right) x^{2} \\
& \left.+7 W_{\star}\left(329 W_{\star}+551\right)+592 x^{2}+2336\right) \\
& +r^{2}\left(-14 W_{\star}\left(x^{2}\left(7 W_{p}\left(245 x^{4}+190 x^{2}-99\right)-2124 x^{2}+12\right)+96\right)\right. \\
& +2 x^{2}\left(7 W_{p}\left(2940 W_{p} x^{6}+\left(3836 W_{p}-7381\right) x^{4}-98\left(20 W_{p}+3\right) x^{2}-112 W_{p}+619\right)\right. \\
& \left.\left.+8\left(2881 x^{2}-680\right)\right)+49 W_{\star}^{2}\left(35 x^{4}+18 x^{2}-5\right)+64\right) \\
& +14 r x\left(-980 W_{p}^{2} x^{6}+W_{p} x^{4}\left(-728 W_{p}+245 W_{\star}+2432\right)+2 x^{2}\left(W_{p}\left(182 W_{p}+63 W_{\star}+8\right)-2\left(77 W_{\star}+317\right)\right)\right. \\
& \left.-35 W_{p} W_{\star}-96 W_{p}-28 W_{\star}+260\right) \\
& \left.+49\left(x^{2}\left(W_{p}\left(35 W_{p} x^{4}+2\left(9 W_{p}-44\right) x^{2}-5 W_{p}-8\right)+52\right)-4\right)\right] \\
& A_{03}=-\frac{5 r^{2}\left(x^{2}-1\right)^{3}(2 r(r-x)+1)\left(r\left(W_{p} x(r-2 x)-r W_{\star} x+W_{\star}\right)+W_{p} x\right)^{2}}{16\left(r^{2}-2 r x+1\right)^{2}}
\end{aligned}
$$$$
A_{13}=\frac{1}{112\left(r^{2}-2 r x+1\right)^{2}}\left[3\left(x^{2}-1\right)^{2}\left(r\left(W_{p} x(r-2 x)-r W_{\star} x+W_{\star}\right)+W_{p} x\right)\right.
$$$$
\times\left(70 r^{6} x\left(7 x^{2}-1\right)\left(W_{p}-W_{\star}\right)+2 r^{5}\left(245 x^{4}\left(W_{\star}-3 W_{p}\right)+x^{2}\left(-105 W_{p}+420 W_{\star}+76\right)-35 W_{\star}+8\right)\right.
$$$$
+r^{4} x\left(x^{2}\left(1715 W_{p}-1015 W_{\star}-304\right)+980 W_{p} x^{4}-7 W_{p}-413 W_{\star}-200\right)
$$$$
+r^{3}\left(x^{2}\left(-644 W_{p}+693 W_{\star}+488\right)-1540 W_{p} x^{4}+63 W_{\star}+58\right)
$$$$
\left.\left.+7 r^{2} x\left(W_{p}\left(123 x^{2}+11\right)-26 W_{\star}-36\right)+14 r\left(-14 W_{p} x^{2}+W_{\star}+3\right)+14 W_{p} x\right)\right],
$$$$
A_{23}=\frac{1}{112\left(r^{2}-2 r x+1\right)^{2}}\left[( x ^ { 2 } - 1 ) \left(-210 r^{8} x^{2}\left(21 x^{4}-14 x^{2}+1\right)\left(W_{p}-W_{\star}\right)^{2}\right.\right.
$$$$
+2 r^{7} x\left(W_{p}-W_{\star}\right)\left(-2205 x^{6}\left(W_{\star}-5 W_{p}\right)-10 x^{4}\left(441 W_{p}+588 W_{\star}+116\right)\right.
$$$$
\left.+3 x^{2}\left(-245 W_{p}+1365 W_{\star}+72\right)-210 W_{\star}+48\right)
$$$$
+r^{6}\left(-5 x^{6}\left(2989 W_{p}^{2}-2 W_{p}\left(5929 W_{\star}+928\right)+W_{\star}\left(2989 W_{\star}+928\right)\right)\right.
$$$$
+2 x^{4}\left(7245 W_{p}^{2}+W_{p}\left(1240-10710 W_{\star}\right)-48 W_{\star}\left(105 W_{\star}+59\right)\right)
$$$$
+3 x^{2}\left(21 W_{p}^{2}-14 W_{p}\left(103 W_{\star}+24\right)+W_{\star}\left(2681 W_{\star}+416\right)-128\right)
$$$$
\left.+17640 W_{p} x^{8}\left(W_{\star}-2 W_{p}\right)+6\left(16-35 W_{\star}\right) W_{\star}+48\right)
$$$$
+2 r^{5} x\left(2 x^{2}\left(-1512 W_{p}^{2}+21 W_{p}\left(271 W_{\star}+17\right)+W_{\star}\left(546 W_{\star}+995\right)+384\right)\right.
$$$$
+8820 W_{p}^{2} x^{8}+x^{4}\left(-21735 W_{p} W_{\star}-4 W_{p}\left(1645 W_{p}+2468\right)+9730 W_{\star}^{2}+7688 W_{\star}\right)
$$$$
\left.+40 W_{p} x^{6}\left(784 W_{p}-637 W_{\star}-116\right)+3\left(W_{p}\left(91 W_{\star}+54\right)-6 W_{\star}\left(91 W_{\star}+29\right)+24\right)\right)
$$$$
+r^{4}\left(-42140 W_{p}^{2} x^{8}+x^{4}\left(4\left(2429 W_{p}-4894\right) W_{\star}+16\left(W_{p}\left(966 W_{p}+841\right)-96\right)-12145 W_{\star}^{2}\right)\right.
$$$$
+2 W_{p} x^{6}\left(-19390 W_{p}+28385 W_{\star}+13056\right)+14 W_{\star} x^{2}\left(-589 W_{p}+65 W_{\star}-10\right)
$$$$
\left.+8\left(W_{p}\left(98 W_{p}-221\right)-240\right) x^{2}+3 W_{\star}\left(161 W_{\star}+76\right)+40\right)
$$$$
+2 r^{3} x\left(x^{2}\left(-2513 W_{p}^{2}+14 W_{p}\left(61 W_{\star}-98\right)+10 W_{\star}\left(182 W_{\star}+597\right)+1440\right)+20055 W_{p}^{2} x^{6}\right.
$$ 


$$
\begin{aligned}
& \left.+W_{p} x^{4}\left(4018 W_{p}-15365 W_{\star}-14208\right)+511 W_{p} W_{\star}+180 W_{p}-196 W_{\star}^{2}-314 W_{\star}+352\right) \\
& +r^{2}\left(-x^{2}\left(-539 W_{p}^{2}+8 W_{p}\left(105 W_{\star}+46\right)+12 W_{\star}\left(35 W_{\star}+291\right)+1952\right)\right. \\
& \left.-19005 W_{p}^{2} x^{6}+2 W_{p} x^{4}\left(413 W_{p}+4060 W_{\star}+7520\right)+4 W_{\star}\left(7 W_{\star}+33\right)-64\right) \\
& +4 r x\left(W_{p}\left(-x^{2}\left(112 W_{p}+210 W_{\star}+971\right)+1120 W_{p} x^{4}+14 W_{\star}+33\right)+98 W_{\star}+140\right) \\
& \left.\left.+28 W_{p} x^{2}\left(-15 W_{p} x^{2}+W_{p}+14\right)-56\right)\right] \text {, } \\
& A_{33}=\frac{1}{112\left(r^{2}-2 r x+1\right)^{2}}\left[14 r^{8} x^{2}\left(21 x^{2}\left(11 x^{4}-15 x^{2}+5\right)-5\right)\left(W_{p}-W_{\star}\right)^{2}\right. \\
& +2 r^{7} x\left(W_{p}-W_{\star}\right)\left(1617 x^{8}\left(W_{\star}-5 W_{p}\right)+21 x^{6}\left(399 W_{p}+175 W_{\star}+52\right)-5 x^{4}\left(147 W_{p}+1323 W_{\star}+176\right)\right. \\
& \left.+x^{2}\left(-455 W_{p}+2065 W_{\star}-12\right)-70 W_{\star}+24\right) \\
& +r^{6}\left(-21 x^{8}\left(119 W_{p}^{2}+2 W_{p}\left(945 W_{\star}+208\right)-W_{\star}\left(553 W_{\star}+208\right)\right)\right. \\
& +x^{6}\left(-23275 W_{p}^{2}+W_{p}\left(47726 W_{\star}+2248\right)+W_{\star}\left(2303 W_{\star}+3456\right)\right) \\
& +x^{4}\left(6055 W_{p}^{2}+W_{p}\left(3664-2030 W_{\star}\right)-21 W_{\star}\left(695 W_{\star}+256\right)+704\right) \\
& +x^{2}\left(119 W_{p}^{2}-6 W_{p}\left(413 W_{\star}+52\right)+W_{\star}\left(4109 W_{\star}+192\right)-528\right) \\
& \left.-12936 W_{p} x^{10}\left(W_{\star}-2 W_{p}\right)-70 W_{\star}^{2}+48 W_{\star}+48\right) \\
& +2 r^{5} x\left(x^{6}\left(20776 W_{p}^{2}+W_{p}\left(8379 W_{\star}+8248\right)-2 W_{\star}\left(4067 W_{\star}+4034\right)\right)\right. \\
& +x^{4}\left(2660 W_{p}^{2}-W_{p}\left(24745 W_{\star}+7297\right)+W_{\star}\left(3094 W_{\star}+2165\right)-1408\right) \\
& -6468 W_{p}^{2} x^{10}+x^{2}\left(4025 W_{p} W_{\star}-2 W_{p}\left(742 W_{p}+333\right)+3654 W_{\star}^{2}+2678 W_{\star}+616\right) \\
& \left.-84 W_{p} x^{8}\left(259 W_{p}-238 W_{\star}-52\right)+189 W_{p} W_{\star}+51 W_{p}-854 W_{\star}^{2}-135 W_{\star}+120\right) \\
& +r^{4}\left(x^{6}\left(-39130 W_{p}^{2}+2 W_{p}\left(7329 W_{\star}-724\right)+W_{\star}\left(11123 W_{\star}+23148\right)+2816\right)\right. \\
& +x^{4}\left(3612 W_{p}^{2}+6 W_{p}\left(3591 W_{\star}+2308\right)-W_{\star}\left(6307 W_{\star}+12354\right)+2816\right) \\
& -x^{2}\left(-434 W_{p}^{2}+2 W_{p}\left(1785 W_{\star}+88\right)+W_{\star}\left(1715 W_{\star}+1888\right)+2312\right) \\
& \left.+33516 W_{p}^{2} x^{10}+2 W_{p} x^{8}\left(8624 W_{p}-24157 W_{\star}-13952\right)+259 W_{\star}^{2}+54 W_{\star}+40\right) \\
& +2 r^{3} x\left(x^{4}\left(7455 W_{p}^{2}-W_{p}\left(7749 W_{\star}+7178\right)-W_{\star}\left(1862 W_{\star}+8065\right)-3168\right)\right. \\
& +x^{2}\left(-917 W_{p}^{2}-W_{p}\left(2149 W_{\star}+2276\right)+14 W_{\star}\left(78 W_{\star}+331\right)+480\right) \\
& \left.-17395 W_{p}^{2} x^{8}+W_{p} x^{6}\left(4585 W_{p}+14357 W_{\star}+17264\right)+245 W_{p} W_{\star}+30 W_{p}+98 W_{\star}^{2}+71 W_{\star}+448\right) \\
& +r^{2}\left(x^{4}\left(-2597 W_{p}^{2}+8 W_{p}\left(609 W_{\star}+1382\right)+490 W_{\star}^{2}+5478 W_{\star}+5344\right)\right. \\
& +x^{2}\left(231 W_{p}^{2}+W_{p}\left(420 W_{\star}+416\right)-12\left(W_{\star}\left(21 W_{\star}+233\right)+160\right)\right) \\
& \left.+18081 W_{p}^{2} x^{8}-W_{p} x^{6}\left(9443 W_{p}+8428 W_{\star}+20880\right)+2\left(3-7 W_{\star}\right) W_{\star}-64\right) \\
& +2 r x\left(2 x^{2}\left(56 W_{p}^{2}-14\left(9 W_{p}+13\right) W_{\star}-769 W_{p}-500\right)\right. \\
& \left.-2352 W_{p}^{2} x^{6}+W_{p} x^{4}\left(1344 W_{p}+490 W_{\star}+3103\right)-14 W_{p} W_{\star}+3 W_{p}+140 W_{\star}+328\right) \\
& \left.+14 x^{2}\left(W_{p}\left(35 W_{p} x^{4}-2\left(9 W_{p}+26\right) x^{2}-W_{p}+20\right)+20\right)-56\right] \text {, } \\
& A_{04}=\frac{35 r^{4}\left(x^{2}-1\right)^{4}\left(r\left(W_{p} x(r-2 x)-r W_{\star} x+W_{\star}\right)+W_{p} x\right)^{2}}{256\left(r^{2}-2 r x+1\right)^{2}}, \\
& A_{14}=-\frac{1}{64\left(r^{2}-2 r x+1\right)^{2}}\left[5 r ^ { 2 } ( x ^ { 2 } - 1 ) ^ { 3 } ( r ( W _ { p } x ( r - 2 x ) - r W _ { \star } x + W _ { \star } ) + W _ { p } x ) \left(7 r^{4} x\left(9 x^{2}-1\right)\left(W_{p}-W_{\star}\right)\right.\right. \\
& +r^{3}\left(-42 W_{p}\left(3 x^{4}+x^{2}\right)+7 W_{\star}\left(17 x^{2}-1\right)+4\right) \\
& \left.\left.+r^{2} x\left(5 W_{p}\left(35 x^{2}+1\right)-68 W_{\star}-8\right)+4 r\left(-20 W_{p} x^{2}+3 W_{\star}+1\right)+12 W_{p} x\right)\right] \text {, }
\end{aligned}
$$




$$
\begin{aligned}
& A_{24}=\frac{1}{128\left(r^{2}-2 r x+1\right)^{2}}\left[3 ( x ^ { 2 } - 1 ) ^ { 2 } \left(35 r^{8} x^{2}\left(33 x^{4}-18 x^{2}+1\right)\left(W_{p}-W_{\star}\right)^{2}\right.\right. \\
& +10 r^{7} x\left(W_{p}-W_{\star}\right)\left(21 x^{4}\left(4 W_{p}+19 W_{\star}\right)+14 x^{2}\left(3 W_{p}-13 W_{\star}+2\right)-462 W_{p} x^{6}+7 W_{\star}-4\right) \\
& +r^{6}\left(5 \left(924 W_{p}^{2} x^{8}+7 x^{4}\left(4\left(7 W_{p}+4\right) W_{\star}-8 W_{p}\left(9 W_{p}+4\right)+153 W_{\star}^{2}\right)\right.\right. \\
& +42 W_{p} x^{6}\left(31 W_{p}-65 W_{\star}\right)+2 W_{\star} x^{2}\left(115 W_{p}-187 W_{\star}+44\right) \\
& \left.\left.+W_{\star}\left(7 W_{\star}-8\right)\right)-10 W_{p}\left(5 W_{p}+8\right) x^{2}+8\right) \\
& +2 r^{5} x\left(10 x^{2}\left(65 W_{p}^{2}+W_{p}\left(68-155 W_{\star}\right)-22 W_{\star}\left(8 W_{\star}+3\right)\right)\right. \\
& \left.-5670 W_{p}^{2} x^{6}+35 W_{p} x^{4}\left(-28 W_{p}+225 W_{\star}+16\right)-85 W_{p} W_{\star}-16 W_{p}+400 W_{\star}^{2}-84 W_{\star}-16\right) \\
& +r^{4}\left(x^{2}\left(-197 W_{p}^{2}+32 W_{p}\left(47 W_{\star}-16\right)+8 W_{\star}\left(146 W_{\star}+137\right)+32\right)\right. \\
& \left.+11235 W_{p}^{2} x^{6}-10 W_{p} x^{4}\left(103 W_{p}+904 W_{\star}+208\right)+8\left(-15 W_{\star}^{2}+W_{\star}+2\right)\right) \\
& +8 r^{3} x\left(-710 W_{p}^{2} x^{4}+W_{p} x^{2}\left(86 W_{p}+334 W_{\star}+179\right)-28 W_{p} W_{\star}+7 W_{p}-22 W_{\star}^{2}-48 W_{\star}-4\right) \\
& +8 r^{2}\left(189 W_{p}^{2} x^{4}-W_{p} x^{2}\left(13 W_{p}+46 W_{\star}+54\right)+W_{\star}^{2}+6 W_{\star}+1\right) \\
& \left.\left.+16 r W_{p} x\left(-12 W_{p} x^{2}+W_{\star}+3\right)+8 W_{p}^{2} x^{2}\right)\right] \text {, } \\
& A_{34}=-\frac{1}{64\left(r^{2}-2 r x+1\right)^{2}}\left[( x ^ { 2 } - 1 ) \left(7 r^{8} x^{2}\left(429 x^{6}-495 x^{4}+135 x^{2}-5\right)\left(W_{p}-W_{\star}\right)^{2}\right.\right. \\
& +2 r^{7} x\left(W_{p}-W_{\star}\right)\left(231 x^{6}\left(18 W_{p}+25 W_{\star}\right)+315 x^{4}\left(2 W_{p}-19 W_{\star}+2\right)-35 x^{2}\left(10 W_{p}-39 W_{\star}+12\right)\right. \\
& \left.-6006 W_{p} x^{8}-35 W_{\star}+30\right)+r^{6}\left(35 x^{4}\left(74 W_{p}^{2}+6 W_{p}\left(29 W_{\star}+8\right)+9\left(4-51 W_{\star}\right) W_{\star}\right)\right. \\
& +5 x^{2}\left(22 W_{p}^{2}+W_{p}\left(96-366 W_{\star}\right)+3 W_{\star}\left(187 W_{\star}-96\right)+24\right)+12012 W_{p}^{2} x^{10} \\
& +21 x^{6}\left(6\left(177 W_{p}+20\right) W_{\star}-30 W_{p}\left(31 W_{p}+8\right)+851 W_{\star}^{2}\right)+462 W_{p} x^{8}\left(31 W_{p}-87 W_{\star}\right) \\
& \left.-35 W_{\star}^{2}+60 W_{\star}-24\right)+2 r^{5} x\left(7 x ^ { 4 } \left(850 W_{p}^{2}+5 W_{p}\left(84-535 W_{\star}\right)\right.\right. \\
& \left.-2 W_{\star}\left(508 W_{\star}+255\right)\right)-17094 W_{p}^{2} x^{8} \\
& +5 x^{2}\left(3\left(55 W_{p}+52\right) W_{\star}-2 W_{p}\left(89 W_{p}+180\right)+1056 W_{\star}^{2}-48\right) \\
& \left.+21 W_{p} x^{6}\left(186 W_{p}+1295 W_{\star}+120\right)+145 W_{p} W_{\star}-12 W_{p}-600 W_{\star}^{2}+342 W_{\star}\right) \\
& +r^{4}\left(5 x^{4}\left(-363 W_{p}^{2}+4928 W_{p} W_{\star}+4 W_{\star}\left(307 W_{\star}+387\right)+96\right)+40299 W_{p}^{2} x^{8}\right. \\
& -7 W_{p} x^{6}\left(3095 W_{p}+5464 W_{\star}+1680\right)-24 W_{\star} x^{2}\left(73 W_{p}+146 W_{\star}+103\right) \\
& +\left(7 W_{p}\left(43 W_{p}+240\right)+528\right) x^{2} \\
& \left.+12 W_{\star}\left(15 W_{\star}-7\right)-32\right)+4 r^{3} x\left(-2 x^{2}\left(63 W_{p}^{2}+W_{p}\left(972 W_{\star}+309\right)+2 W_{\star}\left(85 W_{\star}+252\right)+108\right)\right. \\
& \left.-6286 W_{p}^{2} x^{6}+5 W_{p} x^{4}\left(716 W_{p}+738 W_{\star}+543\right)+78 W_{p} W_{\star}-57 W_{p}+132 W_{\star}^{2}+264 W_{\star}-40\right) \\
& +4 r^{2}\left(x^{2}\left(33 W_{p}^{2}+276 W_{p}\left(W_{\star}+1\right)+6 W_{\star}\left(5 W_{\star}+42\right)+142\right)\right. \\
& \left.+2185 W_{p}^{2} x^{6}-2 W_{p} x^{4}\left(537 W_{p}+370 W_{\star}+618\right)-6 W_{\star}\left(W_{\star}+6\right)+2\right) \\
& +16 r x\left(W_{p}\left(3 x^{2}\left(12 W_{p}+5 W_{\star}+23\right)-100 W_{p} x^{4}-3\left(W_{\star}+3\right)\right)-2\left(3 W_{\star}+5\right)\right) \\
& \left.\left.+24 W_{p} x^{2}\left(W_{p}\left(5 x^{2}-1\right)-4\right)+16\right)\right] \text {, }
\end{aligned}
$$




$$
\begin{aligned}
& A_{44}=\frac{1}{256\left(r^{2}-2 x r+1\right)^{2}}\left[\left(W_{p}-W_{\star}\right)^{2} x^{2}\left(6435 x^{8}-12012 x^{6}+6930 x^{4}-1260 x^{2}+35\right)\right. \\
& \times r^{8}+2\left(W_{p}-W_{\star}\right) x\left(-12870 W_{p} x^{10}+429\left(40 W_{p}+31 W_{\star}\right) x^{8}-924\left(3 W_{p}+25 W_{\star}-2\right) x^{6}\right. \\
& \left.-630\left(4 W_{p}-19 W_{\star}+4\right) x^{4}+70\left(7 W_{p}-26 W_{\star}+12\right) x^{2}+5\left(7 W_{\star}-8\right)\right) r^{7} \\
& +\left(25740 W_{p}^{2} x^{12}+858 W_{p}\left(23 W_{p}-109 W_{\star}\right) x^{10}\right. \\
& +33\left(1363 W_{\star}^{2}+8\left(421 W_{p}+28\right) W_{\star}-448 W_{p}\left(5 W_{p}+1\right)\right) x^{8} \\
& +84\left(405 W_{p}^{2}+\left(168-87 W_{\star}\right) W_{p}-W_{\star}\left(851 W_{\star}+4\right)\right) x^{6} \\
& -70\left(26 W_{p}^{2}+260 W_{\star} W_{p}+3\left(40-153 W_{\star}\right) W_{\star}-8\right) x^{4} \\
& \left.-10\left(374 W_{\star}^{2}-251 W_{p} W_{\star}-296 W_{\star}+W_{p}\left(17 W_{p}+112\right)+48\right) x^{2}+35 W_{\star}^{2}-80 W_{\star}+48\right) r^{6} \\
& +2 x\left(-40326 W_{p}^{2} x^{10}+33 W_{p}\left(984 W_{p}+2115 W_{\star}+224\right) x^{8}\right. \\
& +12\left(1365 W_{p}^{2}+\left(476-7805 W_{\star}\right) W_{p}-2 W_{\star}\left(832 W_{\star}+483\right)\right) x^{6} \\
& -14\left(940 W_{p}^{2}+\left(960-1975 W_{\star}\right) W_{p}-4 W_{\star}\left(508 W_{\star}+171\right)+80\right) x^{4} \\
& +10\left(105 W_{p}^{2}+\left(90 W_{\star}+296\right) W_{p}+12\left(7-88 W_{\star}\right) W_{\star}+64\right) x^{2} \\
& \left.+800 W_{\star}^{2}+64 W_{p}-205 W_{p} W_{\star}-744 W_{\star}+96\right) r^{5} \\
& +\left(105699 W_{p}^{2} x^{10}-12 W_{p}\left(10241 W_{p}+9176 W_{\star}+3248\right) x^{8}+14\left(1415 W_{p}^{2}+96\left(107 W_{\star}+16\right) W_{p}\right.\right. \\
& \left.+8 W_{\star}\left(176 W_{\star}+255\right)+160\right) x^{6}+20\left(337 W_{p}^{2}+8\left(74-277 W_{\star}\right) W_{p}-4 W_{\star}\left(307 W_{\star}+337\right)+88\right) x^{4} \\
& \left.+\left(-397 W_{p}^{2}+1664\left(W_{\star}-2\right) W_{p}+48\left(W_{\star}\left(146 W_{\star}+69\right)-44\right)\right) x^{2}-16\left(W_{\star}-1\right)\left(15 W_{\star}+2\right)\right) r^{4} \\
& +16 x\left(-4638 W_{p}^{2} x^{8}+7 W_{p}\left(806 W_{p}+434 W_{\star}+369\right) x^{6}\right. \\
& -\left(322 W_{\star}^{2}+4\left(905 W_{p}+272\right) W_{\star}+5 W_{p}\left(290 W_{p}+427\right)+300\right) x^{4} \\
& +\left(-2 W_{p}^{2}+\left(942 W_{\star}+81\right) W_{p}+4 W_{\star}\left(85 W_{\star}+242\right)+88\right) x^{2} \\
& \left.-66 W_{\star}^{2}+31 W_{p}-24 W_{p} W_{\star}-120 W_{\star}+52\right) r^{3} \\
& +16\left(1841 W_{p}^{2} x^{8}-W_{p}\left(2115 W_{p}+714 W_{\star}+1378\right) x^{6}\right. \\
& +\left(507 W_{p}^{2}+4\left(185 W_{\star}+289\right) W_{p}+5 W_{\star}\left(7 W_{\star}+66\right)+243\right) x^{4} \\
& \left.-\left(3 W_{p}\left(3 W_{p}+38\right)+118\right) x^{2}-6 W_{\star}\left(23 W_{p}+5 W_{\star}+42\right) x^{2}+3 W_{\star}\left(W_{\star}+6\right)-5\right) r^{2} \\
& +32 x\left(-196 W_{p}^{2} x^{6}+5 W_{p}\left(40 W_{p}+7 W_{\star}+37\right) x^{4}-2\left(10 W_{\star}+3 W_{p}\left(6 W_{p}+5 W_{\star}+23\right)+22\right) x^{2}\right. \\
& \left.\left.+9 W_{p}+3 W_{p} W_{\star}+12 W_{\star}+20\right) r+16\left(35 W_{p}^{2} x^{6}-10 W_{p}\left(3 W_{p}+4\right) x^{4}+3\left(W_{p}\left(W_{p}+8\right)+4\right) x^{2}-4\right)\right]
\end{aligned}
$$

and

$$
\begin{aligned}
& B_{00}=\frac{1}{7\left(r^{2}-2 r x+1\right)}\left[2 r x^{3}\left(7 W_{p}\left(-2 r^{2}+W-2\right)+4 r^{2} W_{\star}\right)+x^{2}\left(7\left(r^{2}+1\right) W_{p}\left(2 r^{2}-W+2\right)-2 r^{2}\left(7 r^{2}+11\right) W_{\star}\right)\right. \\
&+2\left.r\left(17 r^{2}+7\right) W_{\star} x-20 r^{2} W_{\star}\right], \\
& B_{01}= \frac{1}{7\left(r^{2}-2 r x+1\right)^{2}\left(r^{2}+2 r x+1\right)}\left[( x ^ { 2 } - 1 ) \left(4 r^{2}\left(r^{2}+1\right) x^{4}\left(W_{p}\left(11 r^{2}-7 W+7\right)-11 r^{2} W_{\star}\right)\right.\right. \\
&+r^{2}\left(r^{2}+1\right)\left(23 r^{2}+17\right) W_{\star}+8 r^{3} x^{5}\left(W_{p}\left(-11 r^{2}+7 W-7\right)+2 r^{2} W_{\star}\right) \\
&+x^{2}\left(7\left(r^{2}+1\right)^{3} W_{p}^{2}-\left(14 r^{6}+23 r^{4}+28 r^{2}+7\right)\left(r^{2}+1\right) W_{p}+r^{2}\left(14 r^{6}+21 r^{4}-38 r^{2}+11\right) W_{\star}\right) \\
&+2 r x^{3}\left(\left(r^{4}+55 r^{2}+12\right) r^{2} W_{\star}+W_{p}\left(14 r^{6}+23 r^{4}-7\left(r^{2}+1\right)^{2} W_{p}+28 r^{2}+7\right)\right) \\
&\left.\left.-r\left(37 r^{6}+63 r^{4}+45 r^{2}+7\right) W_{\star} x\right)\right],
\end{aligned}
$$




$$
\begin{aligned}
B_{11}= & \frac{3}{7\left(r^{2}-2 r x+1\right)^{2}\left(r^{2}+2 r x+1\right)}\left[4 r^{2}\left(r^{2}+1\right) x^{6}\left(W_{p}\left(-11 r^{2}+7 W-7\right)+11 r^{2} W_{\star}\right)\right. \\
& +x^{2}\left(\left(r^{2}+1\right)\left(19 r^{4}+14 r^{2}+7\right) W-4 r^{2}\left(13 r^{4}+14 r^{2}+7\right) W_{\star}\right) \\
& +3 r^{2}\left(r^{4}-1\right) W_{\star}+8 r^{3} x^{7}\left(W_{p}\left(11 r^{2}-7 W+7\right)-2 r^{2} W_{\star}\right) \\
& -2 r x^{5}\left(\left(r^{4}+63 r^{2}+12\right) r^{2} W_{\star}+W_{p}\left(14 r^{6}+11 r^{4}-7\left(r^{2}+1\right)^{2} W+7\right)\right) \\
& +x^{4}\left(\left(r^{2}+1\right) W_{p}\left(14 r^{6}+11 r^{4}-7\left(r^{2}+1\right)^{2} W+7\right)-r^{2}\left(14 r^{6}+9 r^{4}-82 r^{2}+11\right) W_{\star}\right) \\
& +r x^{3}\left(\left(47 r^{6}+53 r^{4}+21 r^{2}+7\right) W_{\star}-2\left(19 r^{4}+14 r^{2}+7\right) W\right) \\
& \left.+r\left(-3 r^{6}+19 r^{4}+17 r^{2}+7\right) W_{\star} x\right],
\end{aligned}
$$

$$
\begin{aligned}
& B_{02}=\frac{3}{56\left(r^{2}-2 r x+1\right)^{2}\left(r^{2}+2 r x+1\right)}\left[( x ^ { 2 } - 1 ) ^ { 2 } \left(-2 r^{2}\left(r^{2}+1\right)\left(13 r^{2}+7\right) W_{\star}+4 r^{2} x^{4}\left(8 r^{4} W_{\star}-\left(r^{2}+1\right) W_{p}\left(8 r^{2}-7 W\right)\right)\right.\right. \\
& -2 r x^{3}\left(W_{p}\left(2 r^{2}\left(7 r^{4}+2 r^{2}+7\right)-7\left(r^{2}+1\right)^{2} W_{p}\right)+2\left(3 r^{2}+25\right) r^{4} W_{\star}\right) \\
& +8 r^{3} W_{p} x^{5}\left(8 r^{2}-7 W\right)+x^{2}\left(2\left(-7 r^{4}+4 r^{2}+27\right) r^{4} W_{\star}\right. \\
& \left.\left.\left.+W_{p}\left(2 r^{2}\left(7 r^{6}+9 r^{4}+9 r^{2}+7\right)-7\left(r^{2}+1\right)^{3} W_{p}\right)\right)+4 r^{3}\left(10 r^{4}+11 r^{2}+7\right) W_{\star} x\right)\right], \\
& B_{12}=\frac{1}{28\left(r^{2}-2 r x+1\right)^{2}\left(r^{2}+2 r x+1\right)}\left[( x ^ { 2 } - 1 ) \left(6 r^{2}\left(r^{2}+1\right)\left(r^{2}+11\right) W_{\star}\right.\right. \\
& +60 r^{2} x^{6}\left(\left(r^{2}+1\right) W_{p}\left(8 r^{2}-7 W_{p}\right)-8 r^{4} W_{\star}\right)+120 r^{3} W_{p} x^{7}\left(7 W_{p}-8 r^{2}\right)+2 r x^{5}\left(30 r^{6}\left(7 W_{p}+3 W_{\star}\right)\right. \\
& \left.+3 r^{4}\left(314 W_{\star}-5 W_{p}\left(7 W_{p}+44\right)\right)-2 r^{2} W_{p}\left(91 W_{p}+167\right)-105 W_{p}^{2}\right) \\
& +2 r x^{3}\left(-7\left(r^{2}+1\right)^{2} W_{p}^{2}-2\left(201 r^{4}-42 r^{2}+5\right) r^{2} W_{\star}+2\left(63 r^{6}+290 r^{4}+223 r^{2}+56\right) W_{p}\right) \\
& +x^{2}\left(7\left(r^{2}+1\right)^{3} W_{p}^{2}-2\left(63 r^{6}+290 r^{4}+223 r^{2}+56\right)\left(r^{2}+1\right) W_{p}\right. \\
& \left.+2 r^{2}\left(63 r^{6}+587 r^{4}+469 r^{2}+233\right) W_{\star}\right)+x^{4}\left(2 r^{4}\left(105 r^{2}\left(r^{2}-4\right)-869\right) W_{\star}\right. \\
& \left.-\left(r^{2}+1\right) W_{p}\left(210 r^{6}-15 r^{4}\left(7 W_{p}+44\right)-2 r^{2}\left(91 W_{p}+167\right)-105 W_{p}\right)\right) \\
& \left.\left.-4 r\left(33 r^{6}+163 r^{4}+128 r^{2}+28\right) W_{\star} x\right)\right] \text {, }
\end{aligned}
$$

$$
\begin{aligned}
B_{22}= & \frac{1}{56}\left[\frac{1}{r^{4}+r^{2}\left(2-4 x^{2}\right)+1} \times\left(2 W _ { p } x ^ { 2 } \left(7 r^{6}\left(35 x^{4}+10 x^{2}-21\right)-2 r^{4}\left(280 x^{6}+405 x^{4}-866 x^{2}+265\right)\right.\right.\right. \\
& \left.+r^{2}\left(-747 x^{4}+934 x^{2}-355\right)+56\left(4 x^{2}-1\right)\right) \\
& -\frac{1}{\left(r^{2}-2 r x+1\right)^{2}}\left(2 r W _ { \star } ( r x - 1 ) \left(7 r^{4} x\left(35 x^{4}+10 x^{2}-21\right)+r^{3}\left(-280 x^{6}-1055 x^{4}+618 x^{2}+45\right)\right.\right. \\
& \left.\left.\left.+r^{2} x\left(1055 x^{4}+426 x^{2}-473\right)+r\left(-901 x^{4}+142 x^{2}+87\right)+56 x\left(4 x^{2}-1\right)\right)\right)+7 W_{p}^{2}\left(-35 x^{4}+6 x^{2}+5\right) x^{2}\right]
\end{aligned}
$$

$$
\begin{aligned}
B_{13}= & -\frac{3}{8\left(r^{2}-2 r x+1\right)}\left[( x ^ { 2 } - 1 ) ^ { 2 } \left(-14 r^{3} W_{\star} x+2 r W_{p} x^{3}\left(10 r^{2}-W\right)+4 r^{2} W_{\star}\right.\right. \\
& \left.\left.+x^{2}\left(10 r^{4}\left(W_{\star}-W\right)+r^{2}(W-10) W+W_{p}^{2}\right)\right)\right], \\
B_{23}= & -\frac{1}{4\left(r^{2}-2 r x+1\right)}\left[-3\left(5 x^{4}-6 x^{2}+1\right)\left(W_{p}^{2} x^{2}\left(r^{2}-2 r x+1\right)-4 r^{2} W_{\star}(r x-1)\right)\right. \\
+ & 10 r^{2} x\left(7 x^{4}-10 x^{2}+3\right)\left(r\left(W_{p} x(r-2 x)-r W_{\star} x+W_{\star}\right)+W_{p} x\right) \\
& \left.-12 x\left(1-x^{2}\right)\left(r\left(W_{p} x(r-2 x)-r W_{\star} x+W_{\star}\right)+W_{p} x\right)\right],
\end{aligned}
$$




$$
\begin{aligned}
B_{33}= & -\frac{1}{8\left(r^{2}-2 r x+1\right)}\left[\left(35 x^{4}-30 x^{2}+3\right)\left(W_{p}^{2} x^{2}\left(r^{2}-2 r x+1\right)-4 r^{2} W_{\star}(r x-1)\right)\right. \\
& -2 r^{2} x\left(63 x^{4}-70 x^{2}+15\right)\left(r\left(W_{p} x(r-2 x)-r W_{\star} x+W_{\star}\right)+W_{p} x\right) \\
& \left.+8 x\left(3-5 x^{2}\right)\left(r\left(W_{p} x(r-2 x)-r W_{\star} x+W_{\star}\right)+W_{p} x\right)\right] .
\end{aligned}
$$

[1] L. Amendola, C. Quercellini, and E. Giallongo, Mon. Not. R. Astron. Soc. 357, 429 (2005).

[2] D. J. Eisenstein, W. Hu, and M. Tegmark, Astrophys. J. Lett. 504, L57 (1998).

[3] A. Meiksin, M. White, and J. A. Peacock, Mon. Not. R. Astron. Soc. 304, 851 (1999).

[4] C. Blake and K. Glazebrook, Astrophys. J. 594, 665 (2003).

[5] W. Hu and Z. Haiman, Phys. Rev. D 68, 063004 (2003).

[6] T. Matsubara, Astrophys. J. 615, 573 (2004).

[7] R. E. Angulo, C. M. Baugh, C. S. Frenk, R. G. Bower, A. Jenkins, and S. L. Morris, Mon. Not. R. Astron. Soc. 362, L25 (2005).

[8] H.-J. Seo and D. J. Eisenstein, Astrophys. J. 633, 575 (2005).

[9] M. White, Astropart. Phys. 24, 334 (2005).

[10] D. J. Eisenstein, H.-J. Seo, and M. White, Astrophys. J. 664, 660 (2007).

[11] E. Huff, A. E. Schulz, M. White, D. J. Schlegel, and M. S. Warren, Astropart. Phys. 26, 351 (2007).

[12] R. E. Angulo, C. M. Baugh, C. S. Frenk, and C. G. Lacey, Mon. Not. R. Astron. Soc. 383, 755 (2008).

[13] D. J. Eisenstein, I. Zehavi, D. W. Hogg, R. Scoccimarro, M. R. Blanton, R. C. Nichol, R. Scranton, H.-J. Seo, M. Tegmark, Z. Zheng et al., Astrophys. J. 633, 560 (2005).

[14] S. Cole, W. J. Percival, J. A. Peacock, P. Norberg, C. M. Baugh, C. S. Frenk, I. Baldry, J. Bland-Hawthorn, T. Bridges, R. Cannon et al., Mon. Not. R. Astron. Soc. 362, 505 (2005).

[15] N. Padmanabhan, D. J. Schlegel, U. Seljak, A. Makarov, N. A. Bahcall, M. R. Blanton, J. Brinkmann, D. J. Eisenstein, D. P. Finkbeiner, J. E. Gunn et al., Mon. Not. R. Astron. Soc. 378, 852 (2007).

[16] W. J. Percival, R. C. Nichol, D. J. Eisenstein, J. A. Frieman, M. Fukugita, J. Loveday, A. C. Pope, D. P. Schneider, A. S. Szalay, M. Tegmark et al., Astrophys. J. 657, 645 (2007).

[17] T. Okumura, T. Matsubara, D. J. Eisenstein, I. Kayo, C. Hikage, A. S. Szalay, and D. P. Schneider, Astrophys. J. 676, 889 (2008).

[18] X. Xu, A. J. Cuesta, N. Padmanabhan, D. J. Eisenstein, and C. K. McBride, Mon. Not. R. Astron. Soc. 431, 2834 (2013).

[19] L. Anderson, É. Aubourg, S. Bailey, F. Beutler, V. Bhardwaj, M. Blanton, A. S. Bolton, J. Brinkmann, J. R. Brownstein, A. Burden et al., Mon. Not. R. Astron. Soc. 441, 24 (2014).

[20] R. Tojeiro, A. J. Ross, A. Burden, L. Samushia, M. Manera, W. J. Percival, F. Beutler, J. Brinkmann, J. R. Brownstein, A. J. Cuesta et al., Mon. Not. R. Astron. Soc. 440, 2222 (2014).
[21] E. A. Kazin, J. Koda, C. Blake, N. Padmanabhan, S. Brough, M. Colless, C. Contreras, W. Couch, S. Croom, D. J. Croton et al., Mon. Not. R. Astron. Soc. 441, 3524 (2014).

[22] A. J. Ross, L. Samushia, C. Howlett, W. J. Percival, A. Burden, and M. Manera, Mon. Not. R. Astron. Soc. 449, 835 (2015).

[23] S. Alam, M. Ata, S. Bailey, F. Beutler, D. Bizyaev, J. A. Blazek, A. S. Bolton, J. R. Brownstein, A. Burden, C.-H. Chuang et al., Mon. Not. R. Astron. Soc. 470, 2617 (2017).

[24] F. Beutler, H.-J. Seo, A. J. Ross, P. McDonald, S. Saito, A. S. Bolton, J. R. Brownstein, C.-H. Chuang, A. J. Cuesta, D. J. Eisenstein et al., Mon. Not. R. Astron. Soc. 464, 3409 (2017).

[25] M. Takada, E. Komatsu, and T. Futamase, Phys. Rev. D 73, 083520 (2006).

[26] S. Saito, M. Takada, and A. Taruya, Phys. Rev. D 83, 043529 (2011).

[27] L. Guzzo, M. Pierleoni, B. Meneux, E. Branchini, O. Le Fèvre, C. Marinoni, B. Garilli, J. Blaizot, G. De Lucia, A. Pollo et al., Nature (London) 451, 541 (2008).

[28] K. Yamamoto, T. Sato, and G. Hütsi, Prog. Theor. Phys. 120, 609 (2008).

[29] B. A. Reid, L. Samushia, M. White, W. J. Percival, M. Manera, N. Padmanabhan, A. J. Ross, A. G. Sánchez, S. Bailey, D. Bizyaev et al., Mon. Not. R. Astron. Soc. 426, 2719 (2012).

[30] F. Beutler, S. Saito, H.-J. Seo, J. Brinkmann, K. S. Dawson, D. J. Eisenstein, A. Font-Ribera, S. Ho, C. K. McBride, F. Montesano et al., Mon. Not. R. Astron. Soc. 443, 1065 (2014).

[31] L. Samushia, B. A. Reid, M. White, W. J. Percival, A. J. Cuesta, G.-B. Zhao, A. J. Ross, M. Manera, É. Aubourg, F. Beutler et al., Mon. Not. R. Astron. Soc. 439, 3504 (2014).

[32] A. Oka, S. Saito, T. Nishimichi, A. Taruya, and K. Yamamoto, Mon. Not. R. Astron. Soc. 439, 2515 (2014).

[33] C. Hikage and K. Yamamoto, J. Cosmol. Astropart. Phys. 08 (2013) 019.

[34] M. Takada, R. Ellis, M. Chiba, J. E. Greene, H. Aihara, N. Arimoto, K. Bundy, J. Cohen, O. Doré, G. Graves et al., Publ. Astron. Soc. Jpn. 66, R1 (2014).

[35] A. Aghamousa, J. Aguilar, S. Ahlen, S. Alam, L. E. Allen, C. Allende Prieto, J. Annis, S. Bailey, C. Balland et al. (DESI Collaboration), arXiv:1611.00036.

[36] G. J. Hill, K. Gebhardt, E. Komatsu, N. Drory, P. J. MacQueen, J. Adams, G. A. Blanc, R. Koehler, M. Rafal, M. M. Roth et al., in Panoramic Views of Galaxy Formation and Evolution, edited by T. Kodama, T. Yamada, and K. Aoki, Astronomical Society of the Pacific Conference Series 
Vol. 399 (Astronomical Society of the Pacific, San Francisco, 2008), p. 115.

[37] L. Amendola, S. Appleby, A. Avgoustidis, D. Bacon, T. Baker, M. Baldi, N. Bartolo, A. Blanchard, C. Bonvin, S. Borgani et al., Living Rev. Relativity 21, 2 (2018).

[38] D. Spergel, N. Gehrels, C. Baltay, D. Bennett, J. Breckinridge, M. Donahue, A. Dressler, B. S. Gaudi, T. Greene, O. Guyon et al., arXiv:1503.03757.

[39] M. Crocce and R. Scoccimarro, Phys. Rev. D 77, 023533 (2008).

[40] E. T. Vishniac, Mon. Not. R. Astron. Soc. 203, 345 (1983).

[41] J. N. Fry, Astrophys. J. 279, 499 (1984).

[42] M. H. Goroff, B. Grinstein, S.-J. Rey, and M. B. Wise, Astrophys. J. 311, 6 (1986).

[43] Y. Suto and M. Sasaki, Phys. Rev. Lett. 66, 264 (1991).

[44] N. Makino, M. Sasaki, and Y. Suto, Phys. Rev. D 46, 585 (1992).

[45] B. Jain and E. Bertschinger, Astrophys. J. 431, 495 (1994).

[46] F. R. Bouchet, S. Colombi, E. Hivon, and R. Juszkiewicz, Astron. Astrophys. 296, 575 (1995).

[47] R. Scoccimarro and J. A. Frieman, Astrophys. J. 473, 620 (1996).

[48] F. Bernardeau, S. Colombi, E. Gaztañaga, and R. Scoccimarro, Phys. Rep. 367, 1 (2002).

[49] D. Jeong and E. Komatsu, Astrophys. J. 651, 619 (2006).

[50] T. Matsubara, Phys. Rev. D 77, 063530 (2008).

[51] T. Matsubara, Phys. Rev. D 78, 083519 (2008).

[52] A. Taruya, T. Nishimichi, and S. Saito, Phys. Rev. D 82, 063522 (2010).

[53] T. Nishimichi and A. Taruya, Phys. Rev. D 84, 043526 (2011).

[54] A. Taruya, F. Bernardeau, T. Nishimichi, and S. Codis, Phys. Rev. D 86, 103528 (2012).

[55] P. Valageas, T. Nishimichi, and A. Taruya, Phys. Rev. D 87, 083522 (2013).

[56] D. J. Eisenstein, H.-J. Seo, E. Sirko, and D. N. Spergel, Astrophys. J. 664, 675 (2007).

[57] Y. B. Zel'dovich, Astron. Astrophys. 5, 84 (1970).

[58] H.-J. Seo, E. R. Siegel, D. J. Eisenstein, and M. White, Astrophys. J. 686, 13 (2008).

[59] N. Padmanabhan, M. White, and J. D. Cohn, Phys. Rev. D 79, 063523 (2009).

[60] Y. Noh, M. White, and N. Padmanabhan, Phys. Rev. D 80, 123501 (2009).

[61] H.-J. Seo, J. Eckel, D. J. Eisenstein, K. Mehta, M. Metchnik, N. Padmanabhan, P. Pinto, R. Takahashi, M. White, and X. $\mathrm{Xu}$, Astrophys. J. 720, 1650 (2010).

[62] B. D. Sherwin and M. Zaldarriaga, Phys. Rev. D 85, 103523 (2012).

[63] N. Padmanabhan, X. Xu, D. J. Eisenstein, R. Scalzo, A. J. Cuesta, K. T. Mehta, and E. Kazin, Mon. Not. R. Astron. Soc. 427, 2132 (2012).
[64] S. Tassev and M. Zaldarriaga, J. Cosmol. Astropart. Phys. 10 (2012) 006.

[65] M. Schmittfull, Y. Feng, F. Beutler, B. Sherwin, and M. Y. Chu, Phys. Rev. D 92, 123522 (2015).

[66] H.-J. Seo, F. Beutler, A. J. Ross, and S. Saito, Mon. Not. R. Astron. Soc. 460, 2453 (2016).

[67] M. Schmittfull, T. Baldauf, and M. Zaldarriaga, Phys. Rev. D 96, 023505 (2017).

[68] X. Wang, H.-R. Yu, H.-M. Zhu, Y. Yu, Q. Pan, and U.-L. Pen, Astrophys. J. Lett. 841, L29 (2017).

[69] Y. Yu, H.-M. Zhu, and U.-L. Pen, Astrophys. J. 847, 110 (2017).

[70] R. Hada and D. J. Eisenstein, Mon. Not. R. Astron. Soc. 478, 1866 (2018).

[71] S.-F. Chen, Z. Vlah, and M. White, J. Cosmol. Astropart. Phys. 09 (2019) 017.

[72] F. Bernardeau, Astrophys. J. 433, 1 (1994).

[73] P. Fosalba and E. Gaztanaga, Mon. Not. R. Astron. Soc. 301, 503 (1998).

[74] R. Takahashi, Prog. Theor. Phys. 120, 549 (2008).

[75] B. Bose and K. Koyama, J. Cosmol. Astropart. Phys. 08 (2016) 032.

[76] C. Hikage, K. Koyama, and A. Heavens, Phys. Rev. D 96, 043513 (2017).

[77] A. F. Heavens, S. Matarrese, and L. Verde, Mon. Not. R. Astron. Soc. 301, 797 (1998).

[78] R. Scoccimarro, H. M. P. Couchman, and J. A. Frieman, Astrophys. J. 517, 531 (1999).

[79] V. Springel, Mon. Not. R. Astron. Soc. 364, 1105 (2005).

[80] M. Crocce, S. Pueblas, and R. Scoccimarro, Mon. Not. R. Astron. Soc. 373, 369 (2006).

[81] T. Nishimichi, A. Shirata, A. Taruya, K. Yahata, S. Saito, Y. Suto, R. Takahashi, N. Yoshida, T. Matsubara, N. Sugiyama et al., Publ. Astron. Soc. Jpn. 61, 321 (2009).

[82] A. Lewis, A. Challinor, and A. Lasenby, Astrophys. J. 538, 473 (2000).

[83] Note1, fFTW3 at http://www.fftw.org.

[84] P. A. R. Ade, N. Aghanim, M. Arnaud, M. Ashdown, J. Aumont, C. Baccigalupi, A. J. Banday, R. B. Barreiro, J. G. Bartlett et al. (Planck Collaboration), Astron. Astrophys. 594, A13 (2016).

[85] J. J. M. Carrasco, M. P. Hertzberg, and L. Senatore, J. High Energy Phys. 9 (2012) 82.

[86] L. Fonseca de la Bella, D. Regan, D. Seery, and S. Hotchkiss, J. Cosmol. Astropart. Phys. 11 (2017) 039.

[87] N. Kaiser, Mon. Not. R. Astron. Soc. 227, 1 (1987).

[88] D. J. Eisenstein and W. Hu, Astrophys. J. 496, 605 (1998).

[89] I. Mohammed, U. Seljak, and Z. Vlah, Mon. Not. R. Astron. Soc. 466, 780 (2017).

[90] D. Wadekar and R. Scoccimarro, arXiv:1910.02914. 